\title{
Vegetation Ecology of Coastal belt of Khejuri area of Purba Medinipur District with special reference to Hijli coast, West Bengal, India
}

\author{
Debabrata Das ${ }^{1}$ and Manika Das ${ }^{2}$ \\ ${ }^{I}$-Angiospermic Taxonomy and Ecology Laboratory, P.G Deptt. Of Botany, Darjeeling Govt. College, \\ Darjeeling, W.B. (Previously at Central National Herbarium, Botanical Survey of India, Shibpore, Howrah-1) \\ ${ }^{2}$ - Research Scholar, Raja N L Khan Women's College, Midnapore, Paschim Medinipur, West Bengal, India \\ (Previously at Indian Botanic Garden, Botanical Survey of India, Shibpore, Howrah-1)
}

\begin{abstract}
The present article deals with vegetation admixed with populated shoreline elements along with some exotics which broadcast valid and successional change that can develop community as natural kind if managed by local people. The forest department $(F D)$ is engaged with plantation of varied types of elements since two decades ago. The types of vegetation therefore three types as per the present situation either natural or plantation or preliminary natural followed by plantation type. This is due to the potential use of plants for different purposes either for fuel wood production or by other purpose they served. As a coastal line, all the managerial systems always associated with framing or making shelter belt or green belt for multipurpose reasons. The main theme was to sustain species in the present habitats and withstand there against natural calamities like devastating storms, heavy flood or to check the erosion which is annual and reoccurrence type. Therefore, the species planted there as artificial kind is Casuarina equisetifolia along with Acacia auriculoformis and a few strips of Eucalyptus for the sustenance of life. These species used for fuel wood production as well as for protection of the habitats in the upper strand. But the species planted along the shoreline are Sonneratia apetala, Avicennia officinalis, A. marina, A. alba and Prosopis juliflora. Other species they planted the same site are Acacia nilotica, but the species grew there with stunted growth and dynamics of stability was confined. Therefore, the types of special vegetation required here are to check the habitats as well as to develop biodiversity through the growing vegetation and reaching climax formation by halophytes. Though mixed pattern of vegetation found here are mesophytic, xerophytic, hydrophytic, halophytic, halophytic associate type and exotic introduced type. Most of the species grow there as plantation type except a few true halophytes near the High Tide (HT) and Low Tide (LT) area. Ground vegetation of Aleuropus lagopoide, Suaeda maritima and Suaeda monoica cover the disturbed area grazed by cattle which need immediate protection. A general discussion along with vegetation cover of true halophytes both in natural habitat and in plantation stands have been made for critical analysis as preliminary study. A total number of 16 plant species have been critically analyzed along with some potential plants with market demand have been discussed thoroughly. These will generate oxygen for categorically different researchers in the field of biology in near future to manage the ecosystem more usable than the present and will provoke sufficient path to generate income for eco-sustenance of life.
\end{abstract}

KEYWORDS: Khejuri-Hijli, Coastal area to shore, Ecology of Vegetation, Dynamics of Halophytes, Purba Medinipur, Sustainable Development.

\section{INTRODUCTION}

East Midnapore or Purba Medinipur is one of the 18 administrative districts of West Bengal with its headquarters located at Tamluk which was previously called Tamralipta (Bandyopadhyay, 2009). The district was carved out of the erstwhile Medinipur district on January 1, 2002. The district has 4 sub-divisions: Tamluk, Contai, Egra and Haldia (Anonymous, Website). Total area of the district is 430140 lakh Hac. The Purba Medinipur District is geographically located between $21^{\circ} 36^{\prime} 35^{\prime \prime} \mathrm{N}$ and $22^{\circ} 57^{\prime} 10^{\prime \prime} \mathrm{N}$ latitude and $86^{\circ} 33^{\prime}$ $\mathrm{E}^{\prime}$ West and $88^{\circ} 12^{\prime} 40^{\prime \prime} \mathrm{E}$ East longitude and is surrounded by the Bay of Bengal and Balasore District of Orissa State in its South, Paschim Medinipur in its West, Howrah district in the North and South 24 Parganas in the East. On the basis of soil type, rainfall, source of irrigation, the entire Purba Medinipur District has been categorized under 3 Agro-Ecological Situations (AES). The Block/ Taluka wise distribution in the AES is given hereunder but interesting point is that the study site if fall under coastal saline soils (Table1,2). Area studied under the AES is $15.3 \%$ which is very much interesting to study further for different researches because, pattern of diversity of soil and irrigation channels are varied with high tide and low tide even flooded by seawater during monsoon. 
Hijli coastal area is a part of Contai Sub-division of Purba Medinipur District, West Bengal Coast which is fascinating one due to its soil type. Here, degradation both by natural means and manmade cause is lower in compare to the other areas due to boost vegetation cover particularly introduced type along with a small number of patches of natural kind. Coastal stretch of West Bengal with a length of about $350 \mathrm{~km}$ comprises the two districts namely Purba Medinipur (Earstwhile Medinipur) and Dakshin Chabbispargana (South 24 Pgs). It extends over 0.82 million hectare and $220 \mathrm{~km}$ of coastal line. Muddy coast account for $350 \mathrm{~km}$, of which $90 \%$ are treated as marshy zone having halophytic vegetation and their associated flora and fauna; only around 40 $\mathrm{km}$ is considered as sandy belts. It includes two coastal districts namely the South 24 Parganas, supported by Sunderban Mangrove Ecosystem and Midnapore Coast having sand flats and degraded mangrove patch. Indian part of Sunderban comprises Mangrove area (4262 Square Km) slightly more than that (4109 square Km) of highly reclaimed counterpart in Bangladesh. The Coastal belt of Midnapore District represents $27 \%$ of West Bengal of coastal tract $(60 \mathrm{Km})$ extending along the West Bank of Hooghly estuary from New Digha and then curving around Junput, Dadanpatrabar, Khejuri and Haldia on the east to the further north east up to Tamluk or even on the bank of Rupnarayan (Mandal et al, 2013). Hijli area is fall under Khejuri-II community development block. In West Bengal, three seasons are very much pronounced (Pre monsoon, monsoon and post monsoon), each with four months duration and are mainly governed by rainfall and temperature (Chakraborty, et. al., 2010). No Shorea robusta (Sal) vegetation was noticed here though the opposite southern part of the Contai sub-divisonal village Basantia and Saraswatipur under Chalti Gram Panchyat Samiti area having dominant species as Sal (Das, 2013). But here interesting point is that, plantation type is filled with halophyte species which may be a role model to study ecology there with no disturbance and which is not found in sunderban areas of South 24 parganas. In the present study aspects of vegetation along with impact to develop environment better, have been made because no such type of study have been made earlier except a scattered literature found from different field. So, all the types of vegetation along with impact study would encourage the people to conserve the flora and fauna better for their own purpose and also would qualify the environment sustainable and better managed.

All the types of vegetation record would also enhance the knowledge of biology in near future. As for example, Villagers planted different lemons in their own garden in the same site to earn money annually. These are species like Citrus aurantifolia (Patilebu), Citrus jambhiri ( Jamir/Sarbati) and C. lemon (Gandha lebu). The market value may vary from season to season and the cost is amounting Rs. 80-100/- as Indian rupees per hundred pieces. Byers carry these by personal lorry and transport the same to the market nearby or directly to the big city like Kolkata, Cuttack etc. Other products are Drumstick, Bittergourd, Luffa, Trichosanthes and Brinjal etc. But every day they face pathogenic attack by serious pathogens on green vegetables. Other diseases they face are non-pathogenic or physiological kind. The extent of parasitism of the plant differs from place (habitat) to place in the crop fields. The intensity of the infection of diseases may also affect their yield (Ghosh and Das, 1997). The vegetables they used or sold locally are Kalmi sak (Ipomoea aquatica ), Hinche sak (Enhydra fluctuens ), Susni sak (Marselia aquatica), Gime sak (Glenus opposetifolia), Giria or Nona sak (Suaeda maritima), Kanta note (Amaranthus spinosa), Saluk (Nymphaea alba), Kachu (Alocasia/Colocasia spp.), Sajne (Moringa pterigosperma), Dkehi sak (Diplazium sp.), Ghoragima (Mollugo pentaphylla ), Betho sak (Melilotus alba), Chikuni sak (Polygonum plebejum) and Gandhavaduli (Paederia foetida) etc. Weeds of rice field and agricultural fields are species liken Launea sermentosa, Tylophora asthmatica, Sphaeranthus sp., Grangea madraspatna, Polygonum plebejum, Sessilis indica, Chrozophora rottlerii, Ammania baccifera, Eriocaulon quinquangulare, Coenoplexix sp., Gisekia pharnaceoides var. pharnaceoides, Rotalia indica, Acanthus ilicifolius, Clerodendrum inerme, C. infortunatum, Cassia alata, C. tora, Caesalpinia bonduc, Sida cordata, Cropton bonplandianum, Amaranthus spinosus, Phylla nudiflora and Oldenlandia affinis. In social forestry site they planted Acacia auriculoformis, Acacia nilotica, Prosopis juliflora, Glericidia maculate, and a few areas where the planted Cashew (Anacrdium occidentale) along with teak (Tectona grandia) and Mahogany (Swietenia macrophylla). Amount of leaf litter deposition on ground in plantation stand fur away from coast is higher than coastal mangrove plantation. But the site of second type is important because, this site is nutrient recycling site and managed by nature herself. This is due to huge number of aerial roots on the surface of the floor. This study is important because the litters of the mangroves are important during nutrient recycling. This is also supported by a study of Avicennia officinalis L. leaf in laboratory by Dey et al. (2010) in experimental conditions. They identified a total 44 species of micro arthropods from coastal area of Midnapore under varied ecosystems in which all are belonging to seven groups and are interacting with the litter decomposition and recycling of nutrients. So more research is essential for the litter recycling of other species under the genus Sonneretia (Keora) in the same ecosystem under plantation stand of Coastal Purba Medinipur. 


\section{AREA UNDER STUDY}

The study area is fall under the 2 community development blocks of contain Sub-division of Purba Medinipur District, namely Khejuri-I and II. Grma Panchayats of Khejuri -I community development block (CDB)/Panchyat samiti are Birbandar, Heria, Kalagachia, Kamarda, Lakshi and Tikasi. Head quarter of this community development Block is at Kamarda. Similarly the second one i.e. Khejuri-II CDB having 5 Gram Panchayatas (GP) namely, Baratala, Haludbari, Janka, Khejuri and Nijkashba. Geographical area of the first block is 130.52 square kilometer and the second block is 132.77 square kilometer. The area around Khejuri has come up. first century maps project an emerging island in the area which was dumb. Seventeenth century maps show two islands-Khejuri and Hijli. The entire area was submerged under water. Long embankments have been built to keep the saline water away and the land recovered with great effort. In the days of the Nawabs of Bengal, this area had many salt pans. In the early days of the East India Company, the port and the town flourished at Khejuri. As Kolkata grew in importance Khejuri lost its usefulness to the British (Wikipaedia: Khejuri-I and II CDBs, Purba Medinipur, West Bengal.). Vegetation is admixed, and the pattern is varied from place to place because, the site having dry salty pans to wetland and marshy habitats. To study of source and sink of major vegetation products, different eco-habitats have been taken for consideration. To study at market for vegetation products, wild, horticultural or agricultural type, some villages of Khejuri - I and II CDBs have been selected. Both the sites fall under different Agro-Ecological Situations (Table 1,2). Villages and different belts of non agricultural sites were also taken for vegetation study with water channeling systems which disseminates propagules round the year. Some coastal areas of Hijli of Khejuri-II area have been taken for the study of Natural vegetation, plantation stand and grazing land along with some canals, ditches and small ponds. These sites having seasonal vegetation cover which influence the eco-habitat round the year and sustain the life style of the said people. So, People of the local villages and in shops were taken for the present study to know the empirical knowledge or indigenous knowledge they are applying till date for protecting the vegetation. The area depicted in Map-1, have been taken for the ecological research for a period of 2 years, it may be more time consuming depending upon the load of the work.

Table 1. Distribution of Blocks in each AES

\begin{tabular}{|c|l|l|l|}
\hline Sr no & \multicolumn{1}{|c|}{ AES I } & \multicolumn{1}{c|}{ AES II } & \multicolumn{1}{c|}{ AES III } \\
\hline 1 & Tamluk & Nandigram I & Bhagabanpur I \\
\hline 2 & Sahid Matangini & Nandigram II & Bhagabanpur II \\
\hline 3 & Panskura I & Nandigram III & Egra I \\
\hline 4 & Panskura II & Mahishadal & Egra II \\
\hline 5 & Nandakumar & Sutahata & Potashpur I \\
\hline 6 & Moyna & Haldia & Potashpur II \\
\hline 7 & & Kanthi I & \\
\hline 8 & & Kanthi II & \\
\hline 9 & & Kanthi III & \\
\hline 10 & & Ramnagar I & \\
\hline 11 & & Ramnagar II & \\
\hline 12 & & Khejuri I (Study site)* & \\
\hline 13 & & Khejuri II (Study site)** & \\
\hline
\end{tabular}

***: Single and double asterisks indicate study site which fall under Vegetation Ecology Study.

Table 2. Soil types of each AES

\begin{tabular}{|c|l|c|c|c|c|}
\hline Sr no & Type of AES & $\begin{array}{c}\text { No of Blocks/ } \\
\text { Talukas } \\
\text { covered }\end{array}$ & $\begin{array}{c}\text { Geographical } \\
\text { area( lakh Ha) }\end{array}$ & $\begin{array}{c}\text { No of Gram } \\
\text { Panchayats }\end{array}$ & $\begin{array}{c}\text { No of Revenue } \\
\text { Villages }\end{array}$ \\
\hline 1 & $\begin{array}{l}\text { AES- I Loamy } \\
\text { clay soil }\end{array}$ & 6 & 96990 & 72 & 738 \\
\hline 2 & $\begin{array}{l}\text { AES- II Coastal } \\
\text { saline soil }\end{array}$ & 13 & 220800 & 100 & 1421 \\
\hline 3 & $\begin{array}{l}\text { AES- III Clay } \\
\text { Loamy soil } \\
\text { Total }\end{array}$ & 6 & 112350 & 51 & 876 \\
\hline
\end{tabular}

Source: General Description of the District, Purba Medinipur, Chapter-II, from Website. 


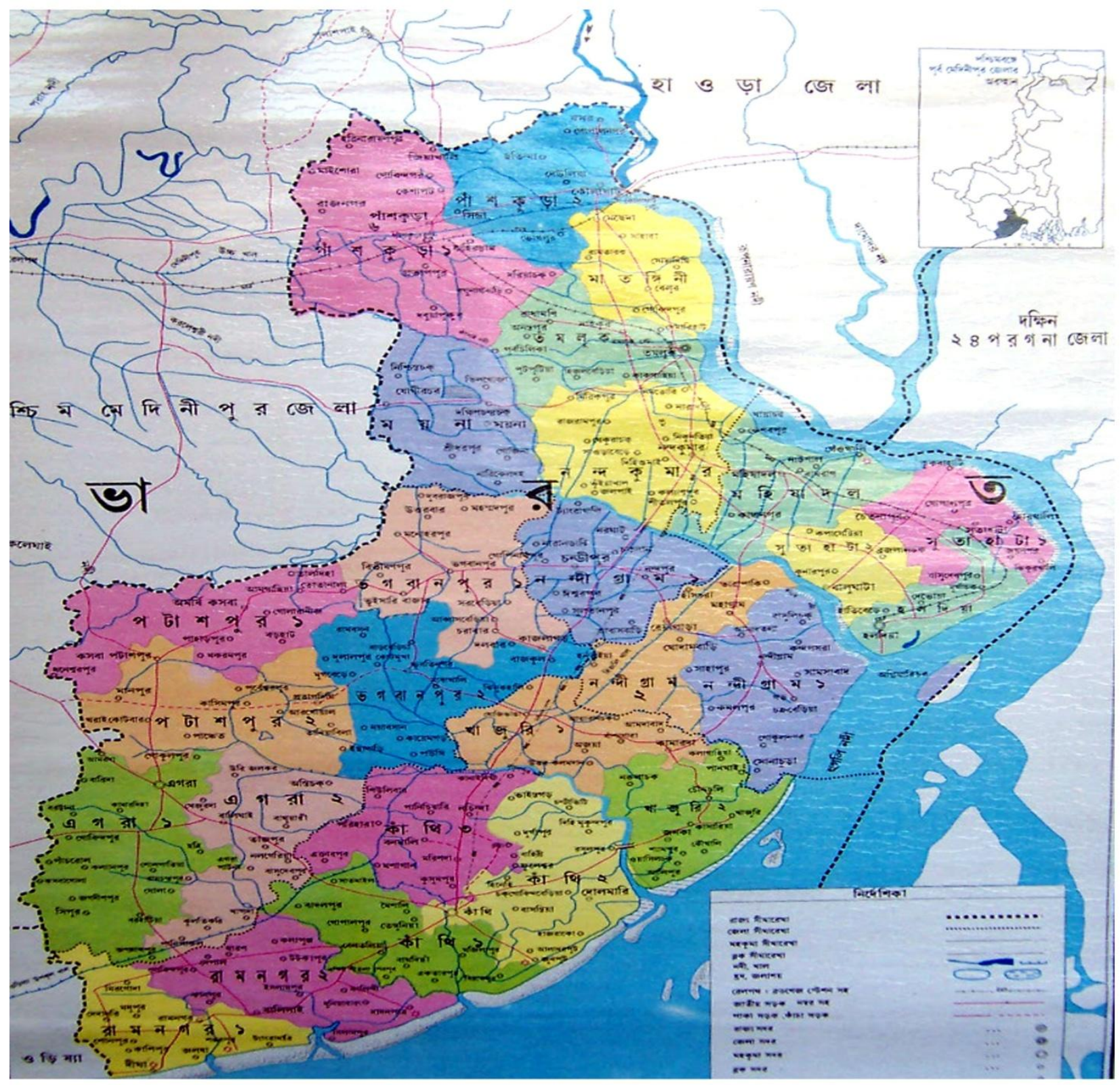

\section{Map 1. Area of study site under Khejuri blocks (I and II) of Purba Medinipur, West Bengal, India: Map taken from Web, Purba Medinipur.}

\section{OBJECTIVES OF STUDY}

Vegetation plays a significant role in protecting sand dunes, alluvial tracts, banks of river, estuary, muddy bundh, bank of ponds, jhills, canals found in coastal area which are eroded seasonally by flood along with High tide and low Tide (HT/LT). Therefore, according to authors, Untawale (1994) and Banerjee (1994) dune vegetation is an ecological storehouse rich in genetic diversity along with high ecological value. Not only that, the species available there are playing a vital role in protecting the coast from erosion and flooding (Desai, 2000). According to Corre Jean-Jacques (1991) coastal sand dunes are the natural structures which protect the coastal environment by absorbing energy from wind, tide, and wave action. In the present study the area with low dune composition but high level of muddy flat basin that broadcasts richness of ground vegetation protect soil havoc to generate succession to reach the vegetation of plantation stand to reach nearer climax. This formation starts with lichens and alga of green type from bed of basin and adjacent mouth of the river. All the species (both herbs and trees) play here a significant role to stabilize habitat and species both flora and fauna rich to richer. Therefore, study and research is essential to come in to a point of following kinds- 
1. To study the vegetation types in Khejuri-I and II Blocks with special reference to Hijli and nearby coast.

2. To study the pattern of Ecosystem dynamics persist in the area.

3. To study the types of vegetation in natural, degraded and plantation sites.

4. To study the flow of fuel wood and related materials collected by the local people.

5. Role of forest departments and public sectors enjoying the scenic beauty along the sustenance of economy of the people for the people and by the people.

6. Study of illegal collection pattern of plant and similar products, and their impact on local vegetation.

7. To prepare Parallel guideline to sustain local vegetation more better through better management of resources.

8. To study ecological status and vegetation and role of Institutions to conserve the environment sound and healthy.

\section{MATERIAL AND METHODS}

Several ecological surveys were done in the villages of Khejuri-I and II community development blocks under Contai sub-division of Purba Medinipur during last year. In this year some surveys of field have been conducted at the coastal areas of Khejuri-II community development block. Survey was conducted in four ways given below: First Way: Field study: Study of vegetation was made at village boundary, roadside, near institutions, near canal bund, near wetlands, wasteland, and in the garden of public sectors of the study area. Study of vegetation was made in open land and in semi-open land as per quadrat methods followed by final checklist of species after seasonal study.

For coastal line special studies were made for Cashew plantation, casuarinas plantation, Acacias and Prosopis plantation including special studies on halophytes of new and old stand with special reference to canopy cover and ground cover., Market study was done for potentially important products of wild and semi wild kind and knowledge taken from resource persons from field regarding their experience to conserve the species of halophytic and non-halophytic type., Second Way: Study of Vegetation: Vegetation study made on the basis of frequency, density and abundance of the species in different stations of the study sites seasonally., Third way: Specimens Collected and studied at laboratory for identification of species and specimens for further research. Soil of different sites were taken for study but till date not analyzed. Some soil of specific sites along with halophytes and halophytic associates were collected for VA-mycorrhizal study. Leaf litter also collected from field for the study of anatomy and morphology of species of special interest to establish stress in such condition. Fourth Way: Indigenous Knowledge Study: Study of Literature and suggestions taken from field workers and villagers to develop further strategies on research and extension activities. Plants are listed alphabetically along with their ecological status including special attention required or not.

Photographs and soils including roots of special interest were collected seasonally to study more and conserve them for further research. All the materials were preserved as Herbarium specimens and museum specimens in our personal custody. 4-5\% copper sulphate solution and 4\% formaldehyde solution were taken for preservation. Field notes including photographs were recorded and preserved for further study. Materials of some plants were also collected from field and preserved in FAA solution. Dry parts of the fruits, seeds, barks and roots were preserved in jars/containers with naphthalene balls. Some photographs were kept in a removable Hard Disk (RHD), and some are under process for publication as recorded one. Some photographs were uploaded in facebook to broadcast the data among the teachers interested in Botany. Underground parts and parts for special interest were kept in the institutional laboratory for future work through the preservatives. Some specimens were under process for the specimens of herbarium section of CAL herb. Identification was done with the help of standard literature (Duthie, 1960; Hooker 1892-1897, Haines, 1921-1925 ; Prain, 1963; Das, 2007, Anonymous, 1997, 2005, 2010, 2012). The names of plants were cross checked and finally placed following Bennet (1987). Previous literature studied for the coastal line research followed by Chakrabiorty et al., (2012). For the identification of species of wetland, Fresh Water Vegetation of Rimer (1984) was followed. To study species of some salty marshes, museum and herbarium section of CAL Herb was used before presenting the document. Herbarium and museum specimens were prepared as per the methodology of Jain and Rao (1977). Different books of Govt. sections have been used to study the use pattern of medicinal plants but for general consideration the common book used was Kirtikar and Basu (1918). Some scattered literature was used to study the Social aspects of plants (Malhotra et al., 1991, Roy et al., 2000). Herbarium specimens collected as per the manual published by Rao and Sharma (1990). Species of Halophyte kind have been identified by the identification manual Mangroves in India (Banerjee et.al., 1986). Other literature made by Blasco (1975), Banerjee (1987), Dwivedi et. al. (1974), Mukherjee (1978), Naskar et. al. (1978), Rao et. al (1972), Sanyal et. al. (1984), Sidhu (1960), Thothatri (1981), Waheed Khan (1959), Walson (1928), Gul and Khan (1995), Subhanian et al. (2010), Jha et. al. (2011), Ahamed et al. (2011), have been consulted for the same purpose. 


\section{RESULTS AND DISCUSSION}

Results revealed that 64 plant species are found there as common occurrence in most of the villages under Khejuri-I and II Community Development blocks. Among them,

Terminalia arjuna (Arjun in Bengali), Spondias pinnata (Amra), Syzygium cumuni (Kalo Jam), Samania saman (Khiris), Diospyros malabarica (Gab), Trewia nodiflora, Thespesia populnea, Casuarina equisetifolia (Jhau), Delonix regia (Gulmohar), Anthocephalus cadamba (Cadam), Acacia nilotica (Babla) and Acacia auriculoformis (Sonajhuri) are used widely as wood of commerce even for fuel wood purpose. The fruits of commercial kind are obtained from species like Zizyphus oenoplea (Kankul), Z. jujube (Topa kul), Moringa oleifera (Sajne/Sanna), Psidium guajava (Piyara), Pithecellobium dulce (Kitchmichi), Mangifera indica (Aam), Cocos nucifera (Narkel), Borassus flabellifer (Tal) and Phoenix sylvestris (Khejur). Medicinal plants of commercial type are Urena lobata, Vitex negundo, Ichnocarpus frutescens, Ambroma augusta, Enhydra fluctuans, Wedelia calandulacea, Glycosmis pentaphylla, Achyranthes aspera and Adhatoda vasica. Species with ornamental value found there are species like Bougainvellia spectabilis (Bagan Bilas), Spathodia campanulata (Tree Tulip), Thevetia peruviana (Kalke) and Anthocephalus cadamba (Kadam) (Table 3).

Table 3. Plant species of Common Occurrence in villages studied under Khejuri-I and II blocks, Contai, Purba Medinipur..

\begin{tabular}{|c|c|c|c|c|}
\hline $\begin{array}{l}\text { Sl. } \\
\text { No. }\end{array}$ & Name & Family & Ecological Notes & $\begin{array}{l}\text { Conservation measures } \\
\text { recommended }\end{array}$ \\
\hline 1. & $\begin{array}{l}\text { Acacia auriculoformis A. Cunn. } \\
\text { Ex Benth. }\end{array}$ & Mimosaceae & $\begin{array}{l}\text { Plantation type, seed } \\
\text { germination rate high. }\end{array}$ & $\begin{array}{l}\text { No need of } \\
\text { conservation, only need } \\
\text { protection during } \\
\text { plantation }\end{array}$ \\
\hline 2. & $\begin{array}{l}\text { Acacia nilotica (L.) Willd. Ex } \\
\text { Del. Ssp. indica (Benth.) Brenan }\end{array}$ & Mimosaceae & Very hardy species & $\begin{array}{l}\text { Not required only need } \\
\text { protection }\end{array}$ \\
\hline 3. & Achras sapoda L. & Sapotaceae & Economic plant & $\begin{array}{l}\text { Spreading through } \\
\text { grafting in nursery }\end{array}$ \\
\hline 4. & Achyranthes aspera L. & Amaranthaceae & $\begin{array}{l}\text { Wild and roots used in } \\
\text { ali (A kind of paste } \\
\text { with turmeric and } \\
\text { Costus rhizome which } \\
\text { is anthelmintic) }\end{array}$ & $\begin{array}{l}\text { Need conservation of } \\
\text { wasteland and village } \\
\text { shrubberies }\end{array}$ \\
\hline 5. & Adhatoda zeylanica Medic. & Acanthaceae & $\begin{array}{l}\text { Propagation through } \\
\text { cuttings }\end{array}$ & $\begin{array}{l}\text { Need conservation of } \\
\text { waste land. }\end{array}$ \\
\hline 6. & Albizia lebbeck (L.) Willd. & Mimosaceae & Tree of commerce & Need protection \\
\hline 7. & $\begin{array}{l}\text { Anthocephalus cadamba (Roxb.) } \\
\text { Mig. }\end{array}$ & Rubiaceae & $\begin{array}{l}\text { Need more plantation } \\
\text { as avenue tree }\end{array}$ & $\begin{array}{l}\text { Need Protection for first } \\
4 \text { years. }\end{array}$ \\
\hline 8. & $\begin{array}{l}\text { Argemone maxicana (Roth.) } \\
\text { Schum. }\end{array}$ & Papaveraceae & $\begin{array}{l}\text { Weeds and seeds are } \\
\text { very dangerous if } \\
\text { mixed in rape seeds. }\end{array}$ & $\begin{array}{l}\text { Need conservation for } \\
\text { biodiversity, but not } \\
\text { truly dangerous, it has } \\
\text { its positive value }\end{array}$ \\
\hline 9. & Streblus asper Lour. & Moraceae & $\begin{array}{l}\text { Wild in village } \\
\text { shrubberies }\end{array}$ & Need conservation \\
\hline 10. & Bambusa bamboos (L.) Voss & Poaceae & Economic and usable & Need plantation \\
\hline 11. & Bambusa tulda Roxb. & Poaceae & Economic and usable & Need more plantation \\
\hline 12. & Bombax ceiba L. & Malvaceae & Flosses are important & $\begin{array}{l}\text { Need potential use in } \\
\text { field for its commercial } \\
\text { value }\end{array}$ \\
\hline 13. & Borassus flabellifer Roxb. & Arecaceae & $\begin{array}{l}\text { Economic as well as } \\
\text { ecologically sound }\end{array}$ & $\begin{array}{l}\text { Need more plantation in } \\
\text { village bund and in } \\
\text { coastal line }\end{array}$ \\
\hline 14. & Bougainvellia spectabilis Willd. & Nyctaginaceae & $\begin{array}{l}\text { Value added for its } \\
\text { ornamental use }\end{array}$ & $\begin{array}{l}\text { Need more cuttings for } \\
\text { developing new types. }\end{array}$ \\
\hline 15. & Caesalpinia bonduc (L.) Roxb. & Caesalpiniaceae & Nuts are economic & $\begin{array}{l}\text { Need protection from } \\
\text { illegal cuttings and } \\
\text { burning. }\end{array}$ \\
\hline
\end{tabular}


Vegetation Ecology of Coastal belt of Khejuri...

\begin{tabular}{|c|c|c|c|c|}
\hline 16. & Calotropis gigantean R. Br. & Asclepiadaceae & $\begin{array}{l}\text { Roots and latex is } \\
\text { economic }\end{array}$ & Need protection \\
\hline 17. & Calotropis procera (Ait.) Ait. f. & Asclepiadaceae & $\begin{array}{l}\text { Flosses are used for its } \\
\text { filling purpose. }\end{array}$ & Need protection \\
\hline 18. & Cassia alata $\mathrm{L}$. & Caesalpiniaceae & Fruits are economic & $\begin{array}{l}\text { No need of } \\
\text { conservation }\end{array}$ \\
\hline 19. & $\begin{array}{l}\text { Casuarina equisetifolia J.R. \& } \\
\text { G. Forst. }\end{array}$ & Casuarinaceae & Economically sound & $\begin{array}{l}\text { Need conservation from } \\
\text { illegal felling and need } \\
\text { protection. }\end{array}$ \\
\hline 20. & Cissus quadrangularis $\mathrm{L}$. & Vitaceae & Stem economic & $\begin{array}{l}\text { Need Protection need } \\
\text { varied use }\end{array}$ \\
\hline 21. & Citrullus colocynthis L. & Cucurbitaceae & Not known & $\begin{array}{l}\text { Used as species for } \\
\text { phytodiversity } \\
\text { conservation }\end{array}$ \\
\hline 22. & $\begin{array}{l}\text { Clerodendrum inerme (L.) } \\
\text { Gaertn. }\end{array}$ & Verbenaceae & $\begin{array}{l}\text { Conserve soil near } \\
\text { bund and in the high } \\
\text { land }\end{array}$ & Need conservation \\
\hline 23. & Cocos nucifera $\mathbf{L}$. & Arecaceaea & Economic & $\begin{array}{l}\text { Highly valued need } \\
\text { protection and need } \\
\text { more plantation }\end{array}$ \\
\hline 24. & Crotalaria pallida Ait. & Fabaceae & Not Known & $\begin{array}{l}\text { Need for species } \\
\text { conservation }\end{array}$ \\
\hline 25. & Croton bonplandianum Baill. & Euphorbiaceae & Anti bleeding agent & $\begin{array}{l}\text { Need protection form } \\
\text { loss }\end{array}$ \\
\hline 26. & Cuscuta reflexa Roxb. & Cuscutaceae & $\begin{array}{l}\text { Febrifuse and used in } \\
\text { viral diseas }\end{array}$ & $\begin{array}{l}\text { Need more study for } \\
\text { research and extension } \\
\text { host diversity study and } \\
\text { chemical analysis is } \\
\text { required. }\end{array}$ \\
\hline 27. & Cynodon dactylon (L.) Pers. & Poaceae & $\begin{array}{l}\text { Fodder plant and sacred } \\
\text { plant for Hindu }\end{array}$ & $\begin{array}{l}\text { Need habitat } \\
\text { conservation to protect } \\
\text { quadruped in villages }\end{array}$ \\
\hline 28. & Cyperus rotunduds L. & Cyperaceae & Fodder plant & Need protection \\
\hline 29. & Delonix regia (Boj.) Raf. & Caesalpiniaceae & Ornamental value & $\begin{array}{l}\text { Need protection from } \\
\text { felling }\end{array}$ \\
\hline 30. & $\begin{array}{l}\text { Diospyros malabarica (Desr.) } \\
\text { Kostel. }\end{array}$ & Ebenaceae & Fruits economic & $\begin{array}{l}\text { Need protection from } \\
\text { felling }\end{array}$ \\
\hline 31. & $\begin{array}{l}\text { Dolichandrone spathacea (L. } \\
\text { f.)K.Schum. }\end{array}$ & Bignoniaceae & $\begin{array}{l}\text { Canal side plant and } \\
\text { frequency is very low }\end{array}$ & $\begin{array}{l}\text { Need special attention } \\
\text { to study. }\end{array}$ \\
\hline 32. & Ficus benghalensis $\mathrm{L}$. & Moraceae & Common but religious & Need Protection \\
\hline 33. & Ficus religiosa L. & Moraceae & Common but religious & Need Protection \\
\hline 34. & Fimbrystylis tetragona $\mathrm{R} . \mathrm{Br}$. & Cyperaceae & $\begin{array}{l}\text { Frequency low need } \\
\text { special attention }\end{array}$ & Need protection \\
\hline 35. & Glycosmis pentaphylla Corr. & Rutaceae & $\begin{array}{l}\text { Common and helps to } \\
\text { study community for } \\
\text { plant population }\end{array}$ & Need special attention \\
\hline 36. & $\begin{array}{l}\text { Ichnocarpus frutescens (L.) } \\
\text { R.Br. }\end{array}$ & Apocynaceae & $\begin{array}{l}\text { Medicinal and } \\
\text { economic }\end{array}$ & $\begin{array}{l}\text { Need special protection } \\
\text { because frequency is } \\
\text { low. }\end{array}$ \\
\hline 37. & Ipomoea fistulosa Mart ex Choisy & Convolvulaceae & $\begin{array}{l}\text { Widely used for } \\
\text { fencing purpose for fuel } \\
\text { wood purpose }\end{array}$ & $\begin{array}{l}\text { Need attention to } \\
\text { increase population }\end{array}$ \\
\hline 38. & Jatropha gossypifolia L. & Euphorbiaceae & $\begin{array}{l}\text { Seeds are economic and } \\
\text { twigs are used as tooth } \\
\text { brushes }\end{array}$ & Need protection \\
\hline 39. & Kyllinga triceps Roth. & Cyperaceae & Economic & $\begin{array}{l}\text { Need habitat } \\
\text { conservation }\end{array}$ \\
\hline 40. & Mangifera indica L. & Anacardiaceae & Mostly economic & Need more plantation \\
\hline
\end{tabular}


Vegetation Ecology of Coastal belt of Khejuri...

\begin{tabular}{|c|c|c|c|c|}
\hline 41. & $\begin{array}{l}\text { Mikania cordata (Burm. f.) } \\
\text { Robinson }\end{array}$ & Asteraceae & Medicinal one & $\begin{array}{l}\text { Need habitat } \\
\text { conservation }\end{array}$ \\
\hline 42. & Moringa oleifera $\mathbf{L}$. & Moringiaceae & $\begin{array}{l}\text { Economic and highly } \\
\text { priced }\end{array}$ & $\begin{array}{l}\text { Need large scale } \\
\text { plantation }\end{array}$ \\
\hline 43. & Oldenlandia stricta L. & Rubiaceae & $\begin{array}{l}\text { Species of low } \\
\text { population }\end{array}$ & Need protection \\
\hline 44. & Opuntia monocantha Haw. & Opuntiaceae & Population insufficient & $\begin{array}{l}\text { Need population } \\
\text { protection }\end{array}$ \\
\hline 45. & Opuntia stricta (Haw.) Haw. & Opuntiaceae & Population low & $\begin{array}{l}\text { Need population } \\
\text { protection }\end{array}$ \\
\hline 46. & Pandanus fascicularis Lam. & Pandanaceae & $\begin{array}{l}\text { Frequency moderately } \\
\text { low }\end{array}$ & $\begin{array}{l}\text { Need Protection of } \\
\text { microclimate }\end{array}$ \\
\hline 47. & Phoenix sylvestris (L.) Roxb. & Arecaceae & Very low population & $\begin{array}{l}\text { Need special attention } \\
\text { to develop or increase } \\
\text { population }\end{array}$ \\
\hline 48. & Phylla nudiflora (L.) Greene. & Euphorbiaceae & Fodder plant & $\begin{array}{l}\text { Frequency High, ionno } \\
\text { need of conservat }\end{array}$ \\
\hline 49. & $\begin{array}{l}\text { Pithecellobium dulce (Roxb.) } \\
\text { Benth. }\end{array}$ & Mimosaceae & Economic & Need protection \\
\hline 37. & Pongamia pinnata Vent. & Fabaceae & $\begin{array}{l}\text { Oil yielding plant, } \\
\text { economic }\end{array}$ & Need more plantation \\
\hline 50. & Prosopis juliflora (Sw. ) DC. & Mimosaceae & Fuel wood species & Need more plantation \\
\hline 51. & Psidium guajava $\mathbf{L}$. & Myrtaceae & Economic & $\begin{array}{l}\text { Need to develop } \\
\text { orchard }\end{array}$ \\
\hline 52. & Ricinus communis L. & Euphorbiaceae & Economic & Need more plantation \\
\hline 53. & Samania saman (Jacq.) Merril & Mimosaceae & Wood of commerce & Need protection \\
\hline 54. & Spathodia campanulata Beauv. & Bignoniaceae & Ornamental tree & Need more plantation \\
\hline 55. & Spondias pinnata (L.f.) Kurz. & Anacardiaceae & Commercial & $\begin{array}{l}\text { Need large scale } \\
\text { cultivation }\end{array}$ \\
\hline 56. & Syzygium cumuni (L.) Skeels & Myrtaceae & Fruits & Wild \\
\hline 57. & $\begin{array}{l}\text { Terminalia arjuna (Roxb.) } \\
\text { Wight. \& Arn. }\end{array}$ & Combretaceae & Medicinal & Need conservation \\
\hline 58. & Thespesia populnea (L.) Soland. & Malvaceae & Economic & Need more plantation \\
\hline 59. & $\begin{array}{l}\text { Thevetia peruviana (Pers.) } \\
\text { Schum. }\end{array}$ & Apocynaceae & $\begin{array}{l}\text { Flowering plants } \\
\text { mostly in gardens }\end{array}$ & $\begin{array}{l}\text { Need attention to spread } \\
\text { the plats }\end{array}$ \\
\hline 60. & Tiliacora acuminata Colebr. & Menispermaceae & Wild woody climber & Need species protection \\
\hline 61. & Trewia nudiflora $\mathbf{L}$. & Euphorbiaceae & $\begin{array}{l}\text { Wood of commerce } \\
\text { used parallel to Gamhar } \\
\text { (Gmelina arborea) }\end{array}$ & $\begin{array}{l}\text { Need more plantation in } \\
\text { roadside. }\end{array}$ \\
\hline 62. & Urena lobata $\mathbf{L}$. & Malvaceae & Medicinal & $\begin{array}{l}\text { Need habitat } \\
\text { conservation }\end{array}$ \\
\hline 63. & Vitex negundo L. & Vitaceae & Medicinal & $\begin{array}{l}\text { Need habitat } \\
\text { conservation }\end{array}$ \\
\hline 64. & Zizyphus oenoplea (L.) Mill. & Rhamnaceae & Economic & Need protection \\
\hline
\end{tabular}

Plant species of commercial importance in village markets are species like Alocasia indica, Colocasia esculenta, Enhydra fluctuans, Glinus oppositifolius, Ipomoea aquatica, Ipomoea batatas, Musa paradisiaca, Portulaca quadrifoliata, Dioscorea alata, Enhydra fluctuans, Chenopodium album, Centella asiatica, Marselia quadrifolia and Polygonum plebejum. The availability of the plants in the weekly markets of the said area varies from season to season. During monsoon the frequency of occurrence is very low in comparison to winter (Table 4). During summer the quality of the products is very good in compare to the products of monsoon. 
Table 4. Plant species of commercial importance in village markets of Study site.

\begin{tabular}{|c|c|c|c|c|}
\hline Sl. No. & Name & Family & Ecological Notes & $\begin{array}{l}\text { Conservation measures } \\
\text { recommended }\end{array}$ \\
\hline 1. & $\begin{array}{l}\text { Alocasia indica (Roxb.) } \\
\text { Schoot. }\end{array}$ & Araceae & $\begin{array}{l}\text { Moderately } \\
\text { abundant }\end{array}$ & $\begin{array}{l}\text { Need aquatic and semi-aquatic } \\
\text { habitat conservation }\end{array}$ \\
\hline 2. & Amaranthus spinosus L. & Amaranthaceae & Low abundance & $\begin{array}{l}\text { Need Wasteland conservation, as } \\
\text { well as conservation of garden } \\
\text { habitat. }\end{array}$ \\
\hline 3. & Amaranthus viridis L. & Amaranthaceae & Low abundance & Need Wasteland conservation \\
\hline 4. & Amorphophalus sylvatica & Araceae & Low abundant & $\begin{array}{l}\text { Need more plantation and need } \\
\text { more species introduction }\end{array}$ \\
\hline 5 . & $\begin{array}{l}\text { Centella asiatica (L.) } \\
\text { Urbn. }\end{array}$ & Apiaceae & $\begin{array}{l}\text { Moderately } \\
\text { abundant }\end{array}$ & $\begin{array}{l}\text { Need restoration of natural eco- } \\
\text { habitat. }\end{array}$ \\
\hline 6. & Chenopodium album $\mathbf{L}$. & Chenopodiaceae & Cropland species & Need cultivation \\
\hline 7. & $\begin{array}{l}\text { Colocasia esculenta (L.) } \\
\text { Schoot }\end{array}$ & Araceae & Highly abundant & $\begin{array}{l}\text { Need special attention to cultivate } \\
\text { in a large scale. }\end{array}$ \\
\hline 8. & Dioscorea alata $\mathrm{L}$. & Dioscoreaceae & Low abundant & $\begin{array}{l}\text { Need more plantation and need } \\
\text { more species introduction }\end{array}$ \\
\hline 9. & Enhydra fluctuans Lour. & Asteraceae & Ecotone species & $\begin{array}{l}\text { Need special attention to develop } \\
\text { more in aquatic but fresh water } \\
\text { habitats }\end{array}$ \\
\hline 10. & $\begin{array}{l}\text { Glinus opposetifolius (L.) } \\
\text { A. Dc. }\end{array}$ & Aizoaceae & $\begin{array}{l}\text { Grown in Moist } \\
\text { and semidry habitat }\end{array}$ & $\begin{array}{l}\text { No need of conservation because } \\
\text { ecological setting is more. }\end{array}$ \\
\hline 11. & Ipomoea aquatica Forsk. & Convolvulaceae & Ecotone species & Only need habitat conservation \\
\hline 12. & Ipomoea batatus (L.) Lam. & Convolvulaceae & $\begin{array}{l}\text { Moderately } \\
\text { abundant }\end{array}$ & Need more cultivation \\
\hline 13. & Marselia quadrifolia L. & Marseliaceae & Aquatic habitat & No need of conservation \\
\hline 14. & Moringa oleifera Lamk. & Moringaceae & $\begin{array}{l}\text { Moderately } \\
\text { abundant }\end{array}$ & $\begin{array}{l}\text { Need more plantation because } \\
\text { demand is high }\end{array}$ \\
\hline 15 . & Musa paradisiaca $\mathbf{L}$. & Musaceae & $\begin{array}{l}\text { Moderately } \\
\text { abundant }\end{array}$ & Need more plantation \\
\hline 16. & $\begin{array}{l}\text { Paederia scandens (Lour.) } \\
\text { merr. }\end{array}$ & Rubiaceae & Shrubs & $\begin{array}{l}\text { Need more increase population } \\
\text { artificially }\end{array}$ \\
\hline 17. & $\begin{array}{l}\text { Polygonum plebejum } \\
\text { R.Br. }\end{array}$ & Polygonaceae & Rice field flora & $\begin{array}{l}\text { Need habitat conservation, } \\
\text { successive boro (Dec-March) rice } \\
\text { cultivation will diminish rapidly } \\
\text { the germination pattern. }\end{array}$ \\
\hline 18. & Portulaca oleracea $\mathbf{L}$. & Portulacaceae & $\begin{array}{l}\text { High land species, } \\
\text { population low }\end{array}$ & $\begin{array}{l}\text { need conservation of veg. } \\
\text { cultivated }\end{array}$ \\
\hline 19. & Portulaca quadrifida L. & Portulacaceae & & \\
\hline 20. & $\begin{array}{l}\text { Spondias pinnata (L. f.) } \\
\text { Kurz }\end{array}$ & Anacardiaceae & Low abundant & Need plantation in high land \\
\hline 21. & $\begin{array}{l}\text { Suaeda maritima var. } \\
\text { australis (R. Br.) Domin }\end{array}$ & Amaranthaceae & Population low & $\begin{array}{l}\text { Need control grazing and } \\
\text { browsing near coast line }\end{array}$ \\
\hline 22. & Tamarindus indica $\mathrm{L}$. & Tamarindaceae & Low abundant & $\begin{array}{l}\text { Need special attention to conserve } \\
\text { species }\end{array}$ \\
\hline 23. & $\begin{array}{l}\text { Typhonium trilobatum } \\
\text { (L.) Schott. }\end{array}$ & Araceae & Low abundant & Need conservation \\
\hline
\end{tabular}

The demand of the local plants of wild kind is high in local markets. Source and sink relation is directly connected via multidirectional ways as the products are community dependant and with a good kith and kin relation among them. Highly priced and demandable species found there is Moringa oleifera (Table 5) and species of demandable kind are Musa paradisiaca, Polygonum plebejum, Spondias pinnata, Tamarindus indica, Typhonium trilobatum, Marselia quadrifoliata, Amorphophalus sylvatica and Colocasia esculenta. The corms of Typhonium rtrilobatum is also marketed for its great demand and used in cancer research (Fig 49). 
Table 5. Market value of vegetables of wild and semi-wild in occurrence

\begin{tabular}{|c|c|c|c|c|}
\hline Sl. No. & Name & Parts Used & $\begin{array}{l}\text { Market Price (Rs. - } \\
\text { Indian) }\end{array}$ & Comment \\
\hline 1. & $\begin{array}{l}\text { Amorphophalus campanulatus } \\
\text { auct. non Blume ex Decne. }\end{array}$ & Corm & 20/- per kg. & Demandable \\
\hline 2. & Averhoeea carambola L. & Fruits & $12 /-\mathrm{Kg}$. & Low Demand \\
\hline 3. & Centella asiatica (L.) Urban. & Whole plant (Dry) & $6-8 /-$ per $\mathrm{kg}$. & Marketed \\
\hline 4. & Chenopodium album $\mathbf{L}$. & Green Veg. & $12-13 . / \mathrm{Kg}$. & Marketed \\
\hline 5. & Colocasia esculenta (L.) Schott & Corm & $20-25 /-\mathrm{Kg}$. & Demandable \\
\hline 6. & Dillenia indica $\mathrm{L}$. & Fruits & 3-4/-Piece & Highly demandable \\
\hline 7. & Dioscorea alata $\mathbf{L}$. & Tuber & $25 /-\mathrm{kg}$. & Demandable \\
\hline 8. & Diplazium sp. & Fronts (Leaves) & $5 /$-per pack & Less demandable \\
\hline 9. & Enhydra fluctuans Lour. & Green twigs & 5/-per $250 \mathrm{gm}$. & Demandable \\
\hline 10. & $\begin{array}{l}\text { Glinus oppositifolius (L.) A. } \\
\text { DC. }\end{array}$ & Green twigs & 10-15/- per Kg. & Demandable \\
\hline 11. & Ipomoea aquatic Forsk. & Twigs & 3/-per pack & Highly demandable \\
\hline 12. & Ipomoea batatas (L.) Lam. & Tuberous Roots & $25 /-\mathrm{Kg}$. & Highly demandable \\
\hline 13. & Marselia quadrifolia L. & Leaves & With twigs, 20/-Kg & Demandable \\
\hline 14. & Moringa oleifera Lamk. & $\begin{array}{l}\text { Leaves, Fls., } \\
\text { Fruits } \\
\text { (Drumsticks) }\end{array}$ & $\begin{array}{l}\text { 6/-, 150/-, 200/-Kg. } \\
\text { (During Feb.) }\end{array}$ & $\begin{array}{l}\text { Highly priced and } \\
\text { demandable }\end{array}$ \\
\hline 15. & Musa paradisiacal L. & Plantain & 2 Pieces-8/- & Demandable \\
\hline 16. & $\begin{array}{l}\text { Paederia scandens (Lour.) } \\
\text { Merrill }\end{array}$ & Gadal/Gandhi & Rs. $5 /$-per $1 / 2 \mathrm{Kg}$. & Low demand \\
\hline 17. & Polygonum plebejum R. Br. & Twigs as Chikuni & 20/-Kg. & Demandable \\
\hline 18. & Spondias pinnata (L. f.) Kurz & Fruits & $10-15 /-\mathrm{Kg}$. & Demandable \\
\hline 19. & Tamarindus indica $\mathrm{L}$. & $\begin{array}{l}\text { Twigs, green raw } \\
\text { fruits }\end{array}$ & $15 /-, 20 /-\mathrm{Kg}$. & Demandable \\
\hline 20. & $\begin{array}{l}\text { Typhonium trilobatum }(\mathrm{L} .) \\
\text { Schoot }\end{array}$ & $\begin{array}{l}\text { Leaves with } \\
\text { petiole }\end{array}$ & 6 Pieces@ @s.3/- & Demandable \\
\hline
\end{tabular}

Plants of Research Importance in Physiologically dry soil and in sandy beach area are some halophytes. These are Sonneretia apetala (Keora), Salicornia herbacea (Penisak), Suaeda monoica and S. maritima. Fern found there as common one is species like Acrostichum aureum. Fodder grass found there is Aleuropus lagopodides. Sand binder species found there are Ipomoea pes-capre, Salsola kalitenuifolia, Porteresia coarctata, Launea sermentosa, Hydrophylax maritima, Gisekia pharnacoides . Halophytic associates are species like Excoicaria agallocha (Geo) and Acanthus ilicifolius (Hargoja). Species of rare occurrence is Acanthus volubilis. Common cactus found there is Opuntia monocantha (Table 6).

Table 6. Plants of Research Importance in connection with Ecology at the habitat of Physiologically dry soil.

\begin{tabular}{|l|l|l|l|l|}
\hline Sl. No. & Plants/Name & Family & Ecological note & Important Notes \\
\hline 1. & $\begin{array}{l}\text { Acanthus ilicifolius L. } \\
\text { (Harkatch/Hargoja) }\end{array}$ & Acanthaceae & $\begin{array}{l}\text { Common in coastal } \\
\text { canals }\end{array}$ & $\begin{array}{l}\text { Gregarious aside the } \\
\text { ecotone ridge of tidal } \\
\text { swamp. }\end{array}$ \\
\hline 2. & $\begin{array}{l}\text { Acanthus volubilis Wall. (Lata } \\
\text { Harkatch) }\end{array}$ & Acanthaceae & Rare in occurrence & $\begin{array}{l}\text { Twining shrub now } \\
\text { rare species along the } \\
\text { sea coast joined with } \\
\text { canal. }\end{array}$ \\
\hline 3. & Acrostichum aureum L. & Pteridaceae & Widely distributed & $\begin{array}{l}\text { Need shrubby and tree } \\
\text { vegetation }\end{array}$ \\
\hline 4. & Aleuropus lagopodides (L.) Trin & Poaceae & & \\
\hline 5. & $\begin{array}{l}\text { Arthrocenemum indicum (Willd.) } \\
\text { Moq. }\end{array}$ & Chenopodiaceae & & $\begin{array}{l}\text { Lanceolate-acute } \\
\text { leaves and narrow - } \\
\text { ellipsoid fruits }\end{array}$ \\
\hline 6. & $\begin{array}{l}\text { Avicennia alba Bl. } \\
=\text { A. officinalis var. alba Cl. (Dulia } \\
\text { Bain) }\end{array}$ & & $\begin{array}{l}\text { Rliptic oblong to } \\
\text { elliptic ovate leaves }\end{array}$ \\
\hline 7. & $\begin{array}{l}\text { Avicennia marina var. marina } \\
\text { (Fors.) Vierh. (Boro Baen) }\end{array}$ & & is apioneer species \\
\hline
\end{tabular}




\begin{tabular}{|c|c|c|c|c|}
\hline & & & $\begin{array}{l}\text { towards sea-ward } \\
\text { areas subjected to } \\
\text { high wave action, } \\
\text { high salinity, and silt } \\
\text { deposition and } \\
\text { stabilizes intertidal } \\
\text { land mass. }\end{array}$ & \\
\hline 8. & $\begin{array}{l}\text { Avicennia officinalis L. (Kalo } \\
\text { Baen) }\end{array}$ & Avicenniaceae & Low population & $\begin{array}{l}\text { Large sized tree, } \\
\text { obovate to ovate- } \\
\text { oblong leaves. }\end{array}$ \\
\hline 9. & $\begin{array}{l}\text { Bulbostylis barbata (Rottb.) C.B. } \\
\text { Cllarke (Phulur) }\end{array}$ & Cyperaceae & Moderately abundant & Herbs \\
\hline 10. & Excoicaria agallocha L. (Geoan) ) & Euphorbiaceae & Common & Tree \\
\hline 11. & Gisekia pharnaceoides L. (Salup) & Azoiaceae & Common & Herb \\
\hline 12. & Hydrophylax maritima L. (Comir) & Rubiaceae & Less common & Herb \\
\hline 13. & Ipomoea pes-capre (L.) R.Br. & Convolvulaceae & Common & Climber \\
\hline 15. & $\begin{array}{l}\text { Launea sementosa (Willd.) Schult. } \\
\text {-Bip.ex O. Kuntze (Halde) }\end{array}$ & Asteraceae & Common & Herb \\
\hline 14. & $\begin{array}{l}\text { Opuntia monocantha Haw. } \\
\text { (Fanimansa) }\end{array}$ & Opuntiaceae & Less abundant & Shrub \\
\hline 15. & $\begin{array}{l}\text { Porteresia coarctata (Roxb.) } \\
\text { Tateoka (Dhanighas) }\end{array}$ & Poaceae & Less abundant & Herb \\
\hline 16. & Salicornia herbacea L. (Saltwort) & Chenopodiacaea & Mineral rich plant & Herb \\
\hline 17. & Salsola kaliteneuifolia & & & Herb \\
\hline 18. & $\begin{array}{l}\text { Sonneretia apetala Buch. -Ham. } \\
\text { (Keora) }\end{array}$ & Sonneretiaceae & $\begin{array}{l}\text { Previously it was } \\
\text { widespread but now it } \\
\text { is near threatened }\end{array}$ & $\begin{array}{l}\text { Narrowly elliptic } \\
\text { leaves, apetalous } \\
\text { flowers. }\end{array}$ \\
\hline 19. & $\begin{array}{l}\text { Suaeda monoica Forsk. Ex Gmel. } \\
\text { (Nunia) }\end{array}$ & Chenopodiaceae & Salinity indicator. & Small herb \\
\hline 20. & $\begin{array}{l}\text { Suaedia maritime var. australis } \\
\text { (R.Br.) Domin (Nonasak) }\end{array}$ & Amaranthaceae & Salinity indicator & Herb \\
\hline
\end{tabular}

There are 27 medicinal plants found which are potentially important in the shrubberies, gardens, bund of Ponds and Canals, even in protected sites of Govt. and semi-Govt. Institutes and in wasteland. Medicinal plants of potential importance are species like Adhatoda vasica, Aloe vera, Ambroma augusta, Andrographis paniculata, Azadirachta indica, Bryophyllum calycinum, Catharanthus roseus, Centella asiatica, Cissus quadrangularis, Clerodendum serratum, Costus speciosus, Emblica officinalis, Enhydra fluctuans, Euphorbia nerifolia, Gloriosa superba, Ichnocarpus frutescens, Jatropha gossypifolia, Marselia quadrifolia, Ocimum sanctum, Plumbago zeylanica, Smilax ovalifolia, Stephania japonica, Swietenia macrophylla, S. mahagoni, Tylophora tenuis and Wedelia calandulacea (Table 7).These are used locally even are widely used by common people in different forms. These are also marketed outside the area and marketed by stack holders. These products are marketed and channelized to the nodal markets for their company based value. Pharmaceutical companies use the products like dry Centella asiatica plant; dry Ichnocarpus frutescens plants, Gloriosa superba roots and plant materials of Plumbago zeylanica.

Table 7. Some medicinal Plants of potential importance from Khejuri area of Purba Medinipur

\begin{tabular}{|l|l|l|l|l|}
\hline $\begin{array}{l}\text { Sl. } \\
\text { No. }\end{array}$ & Name & Family & Importance & Habitat \\
\hline 1. & Adhatoda vasica Nees & Acanthaceae & Leaves & $\begin{array}{l}\text { Hedge and } \\
\text { Garden }\end{array}$ \\
\hline 2. & Aloe vera Tourn. ex L. & Liliaceae & Leaves & Garden \\
\hline 3. & Ambroma augusta L.f. & Sterculiaceae & Flowers and seeds & Hedge \\
\hline 4. & $\begin{array}{l}\text { Andrographis paniculata (Burm.f.) } \\
\text { Wall ex Nees }\end{array}$ & Acnthaceae & Leaves & Shrubberies \\
\hline 5. & Azadirachta indica A. Juss. & Meliaceae & Fruits, bark and leaves & Pond side \\
\hline 6. & Bryophyllum calycinum Salisb. & Crassulaceae & Leaves & $\begin{array}{l}\text { Garden and } \\
\text { waste land }\end{array}$ \\
\hline 7. & Catharanthus roseus (L.) G. Don. & Apocynaceae & Leaves & Wasteland \\
\hline 8. & Centella asiatica (L.) Urban. & Apiaceae & Leaves & $\begin{array}{l}\text { Pond and } \\
\text { wetland even } \\
\text { in rice field }\end{array}$ \\
\hline 9. & & & & Jungle \\
\hline
\end{tabular}


Vegetation Ecology of Coastal belt of Khejuri...

\begin{tabular}{|c|c|c|c|c|}
\hline 10. & Clerodendrum serratum Spreng. & Verbenaceae & Roots & Shrubberies \\
\hline 11. & Costus speciosus Koen. Ex Retz. & Costaceae & Rhizome & Shrubberies \\
\hline 12. & Emblica officinalis Gaertn. & Euphorbiaceae & Fruits & Garden \\
\hline 13. & Enhydra fluctuans Lour. & Asteraceae & Twigs & $\begin{array}{l}\text { Pond side and } \\
\text { in water of } \\
\text { wet land }\end{array}$ \\
\hline 15. & Euphorbia antiquorum L. & Euphorbiaceae & Roots & Shrubberies \\
\hline 14. & Euphorbia nerifolia L. & Euphorbiaceae & Leaves & $\begin{array}{l}\text { Sacred place } \\
\text { of home } \\
\text { garden }\end{array}$ \\
\hline 15. & Gloriosa superb L. & Liliaceae & $\begin{array}{l}\text { Tuber, roots, Leaves and } \\
\text { Flowers }\end{array}$ & $\begin{array}{l}\text { Shrubberies } \\
\text { and road side } \\
\text { waste land. }\end{array}$ \\
\hline 16. & Ichnocarpus frutescens R. Br. & Apocynaceae & Roots & Shrubberies \\
\hline 17. & Jatropha gossypifolia L. & Euphorbiaceae & Twigs, stems & Road side \\
\hline 18. & Marselia quadrifolia L. & Marseliaceae & Leaves & Aquatic body \\
\hline 19. & Ocimum sanctum L. & Lamiaceae & Leaves & $\begin{array}{l}\text { Home } \\
\text { Garden, } \\
\text { Burning place }\end{array}$ \\
\hline 20. & Plumbago zeylanica L. & Plumbaginaceae & Roots & Jungle \\
\hline 21. & Smilax ovalifolia Roxb. & Smilacaceae & Roots & Shrubberies \\
\hline 22. & Stephania japonica (Thumb.) Miers. & Menispermaceae & Leaves & $\begin{array}{l}\text { Jungle and } \\
\text { waste land }\end{array}$ \\
\hline 23. & Swietenia macrophylla King. & Meliaceae & Seed/Fruit & Plantation site \\
\hline 24. & Swietenia mahagony L. & & & Plantation site \\
\hline 25. & Tylophora tenuis Bl. & Asclepiadaceae & Roots & $\begin{array}{l}\text { Highland of } \\
\text { Coastal strip. }\end{array}$ \\
\hline 26. & Vitex negundo L. & Verbenaceae & Leaves & Shrubberies \\
\hline 27. & $\begin{array}{l}\text { Wedelia calandulacea Less. Non } \\
\text { Rich. }\end{array}$ & Asteraceae & Leaves & Wetland site \\
\hline
\end{tabular}

A setting of ecology near the estuary is also placed here. In old plantation stands, Keora (Sonneratia sp.) plant is common. But the fact is that, the types of plantation are irregular where number of poles per $10 \mathrm{~m} \times 10 \mathrm{~m}$ quadrat varies from 16 to 18 with the variation of one microsite to another. Minimum girth at breast height $(\mathrm{GBH})$ in each quadrat varies from 11 to 12 whereas, highest gbh value varies from 55 to $56 \mathrm{~cm}$ (Table 8). The canopy is two layerd i.e. $1^{\text {st }}$ layer canopy for developing plants whereas, $2^{\text {nd }}$ layer canopy is for ill developed plants. Ground is almost free from vegetation except a few newly settled Acanthus ilicifolius plantlets. Canopy cover is more than 50 percent, so quality of the vegetation growth in good or better not best (Maity et al., 2011). Some sites having a few numbers of felled poles that indicate management is not so good. Therefore, it indicates illegal felling is going on (See cut pole in figure where the author is observing/monitoring the vegetation).

Table 8 Girth at breast height (GBH) values of Keora trees in $10 \mathrm{~m} \times 10 \mathrm{~m}$ quadrats at old plantation stand of Halophytes.

\begin{tabular}{|l|l|l|l|l|}
\hline $\begin{array}{l}\text { No. of Pole per 10m } \\
\text { x10m quadrat }\end{array}$ & GBH of Trees in Q1 & GBH of Trees in Q2 & GBH of Trees in Q3 & GBH of Trees in Q4 \\
\hline In Q1=17 Nos. & $40,25,21,28,44, \mathbf{1 1}$, & $23, \mathbf{1 6}, 28,43,20,34$, & $43,20,34,56,34,28$, & $23,20,34,46,34,28$, \\
In Q2=16 Nos. & $23,55,36,33,40,30$, & $56,34,28,21,40,39$, & $21,40,39,39,22,21$, & $22,40,29,39,22,21$, \\
In Q3=17 Nos. & $43,49,43,20,41=17$ & $39,22,21,27$ & $27,11,21,24,26=17$ & $26, \mathbf{1 2}, 21,24,25$, \\
In Q4=18 Nos. & & $=16$ & & \\
& & & & \\
\hline
\end{tabular}

Note: Q1=Quadrat 1, Q2=Quadrat 2, so on....., Canopy cover is $60 \%$ as per the Graph paper method, GBH in $\mathrm{cm}$.., Bold number indicates lowest GBH value in each quadrat.

Floral elements as Mangrove associates found there are tree species like Thespesia populnea, Barringtonia racemosa, whereas bushy shrubs are Caesal[pinia bonduc and Pandanus odoratissimus. Shrubs as mangrove associates are species like clerodendrum inerme, pluchea alba. Herbs found in the same site, are species like Merope angulata, Salacia chinensis etc. (Table 9). A special twiner i.e. Cannavelia rosea is also found there which is found with Ipomoea pes-capre in sea shore. 
Vegetation Ecology of Coastal belt of Khejuri...

Table 9 Mangrove associates in Khejuri area of Purba Medinipur, West Bengal, India

\begin{tabular}{|c|c|c|c|c|}
\hline Sl. & Name & Family & Habit & Habitat \\
\hline 1. & Barringtonia racemosa Roxb. & Baringtoniaceae & Small tree & $\begin{array}{l}\text { Near bank of river } \\
\text { and pond }\end{array}$ \\
\hline 2. & Cannavelia rosea (Sw.) DC. & Fabaceae & Twiner & Near bank of river \\
\hline 3. & Caesalpinia bonduc (L.) Roxb. & Caesalpiniaceae & Bushy shrub & $\begin{array}{l}\text { Shrubberies, } \\
\text { Coastal bund }\end{array}$ \\
\hline 4. & Clerodendrum inerme Gaertn. & Verbenaceae & Shrub & $\begin{array}{l}\text { Wasteland, } \\
\text { Degraded land }\end{array}$ \\
\hline 5. & $\begin{array}{lll}\begin{array}{l}\text { Fimbrystylis } \\
\text { vahl. }\end{array} & \text { ferruginea } & \text { (L.) } \\
\end{array}$ & Cyperaceae & Small shrub & Coastal canalside. \\
\hline 6. & Ipomoea tuba (Schl.) G. Don & Convolvulaceae & Creeper & Coastal dyke \\
\hline 7. & $\begin{array}{l}\text { Merope angulata } \\
\text { Swingle }\end{array}$ (Willd.) & Rutaceae & Herb & Coastal canal side \\
\hline 8. & Pandanus odoratissimus L. f. & Pandanaceae & Bushy Shrub & $\begin{array}{l}\text { Coastal area and in } \\
\text { rice field. }\end{array}$ \\
\hline 9. & Pluchea alba Less. & Asteraceae & Shrub & Garden side \\
\hline 10. & Salacia chinensis L. & Hypocrataceae & Herb & Near river bank \\
\hline 11. & $\begin{array}{l}\text { Stenochlaena palustre (Burm.) } \\
\text { Bedd. }\end{array}$ & Polypodiaceae & Under shrub & $\begin{array}{l}\text { Underneath of } \\
\text { Canal shrubberies. }\end{array}$ \\
\hline 12. & $\begin{array}{l}\text { Stictocardia } \\
\text { Hall.f. }\end{array}$ & Convolvulaceae & Twiner & Cnal shrubberies \\
\hline 13. & $\begin{array}{l}\text { Thespesia populnea (L.) Sol. Ex } \\
\text { Correa }\end{array}$ & Malvaceae & Tree & $\begin{array}{l}\text { Planted as in garden } \\
\text { and bank of Pond }\end{array}$ \\
\hline 14. & Tylophora tenuis Bl. & Asclepiadaceae & Twiner & $\begin{array}{l}\text { Coastal dyke and in } \\
\text { jungle. }\end{array}$ \\
\hline
\end{tabular}

The study revealed that, the present study sites need immediate protection of some key stone species. These are Tylophora tenuis, Stictocardia tillifolia and Salacia chinensis. For the study of microclimate, these species are essential along with nutrients cycling of the species in the same site. Other species of shrubberies habitat, need protection with the special attention of the community as in- situ type. Species like Ichnocarpus frutescens R. Br. (A species of low occurrence need immediate protection because this species is used in various ways and need local protection), Phoenix sylvestris Roxb. (Leaves used in thatching purpose and even to prepare broom need immediate protection as a fruit tree even producing molasses), Zizyphus oenoplea Mill. and Syzygium cumuni Skeels need immediate and urgent protection to enhance the ecosystem sound due to their all great range of importance. Typhonium trilobatum need special attention to conserve the same in the present habitat, because it is now-a -days used in cancer research.

\section{COMMENTS ON THE PRESENT RESEARCH:}

The present result also revealed that, the scenario of halophytes, halophytic associates in the basin of river Rasulpur and lower part of Haldi, Runarayan and the main flow Hooghly which have potential importance. It would obviously highlight the pattern and dynamics of vegetation and life style of people. This will encourage the persons for research and study of the students and researchers in the field of geography, Environmental Science, Botany, Zoology, management and natural science in near future. The result is fit with the result of publications made by Das and Ghosh (1999) under the topic Mangroves and other phanerogams growing at Nayachar, Haldia, Midnapore, West Bengal. Here no Phoenix palludosa is observed along with Rhizophora, Tamarix, Xylocarpus, Nipa, Heritiera, Ceriops, Bruguiera and Aegiceros. Therefore, for further research and invention about the interaction of newly introduced species, programme may be made. It will generate the long term process of studies through extension as a second step, which may enhance the knowledge the forest department even nursery men to reach their knowledge. Later on the empiric knowledge will gregariously develop economy for the rural people by applying the scientific knowledge and technique in the field of market in near future to develop society more sustainable than the present. 


\section{PHOTOGRAPHS IN PHOTOPLATES (1-4)}

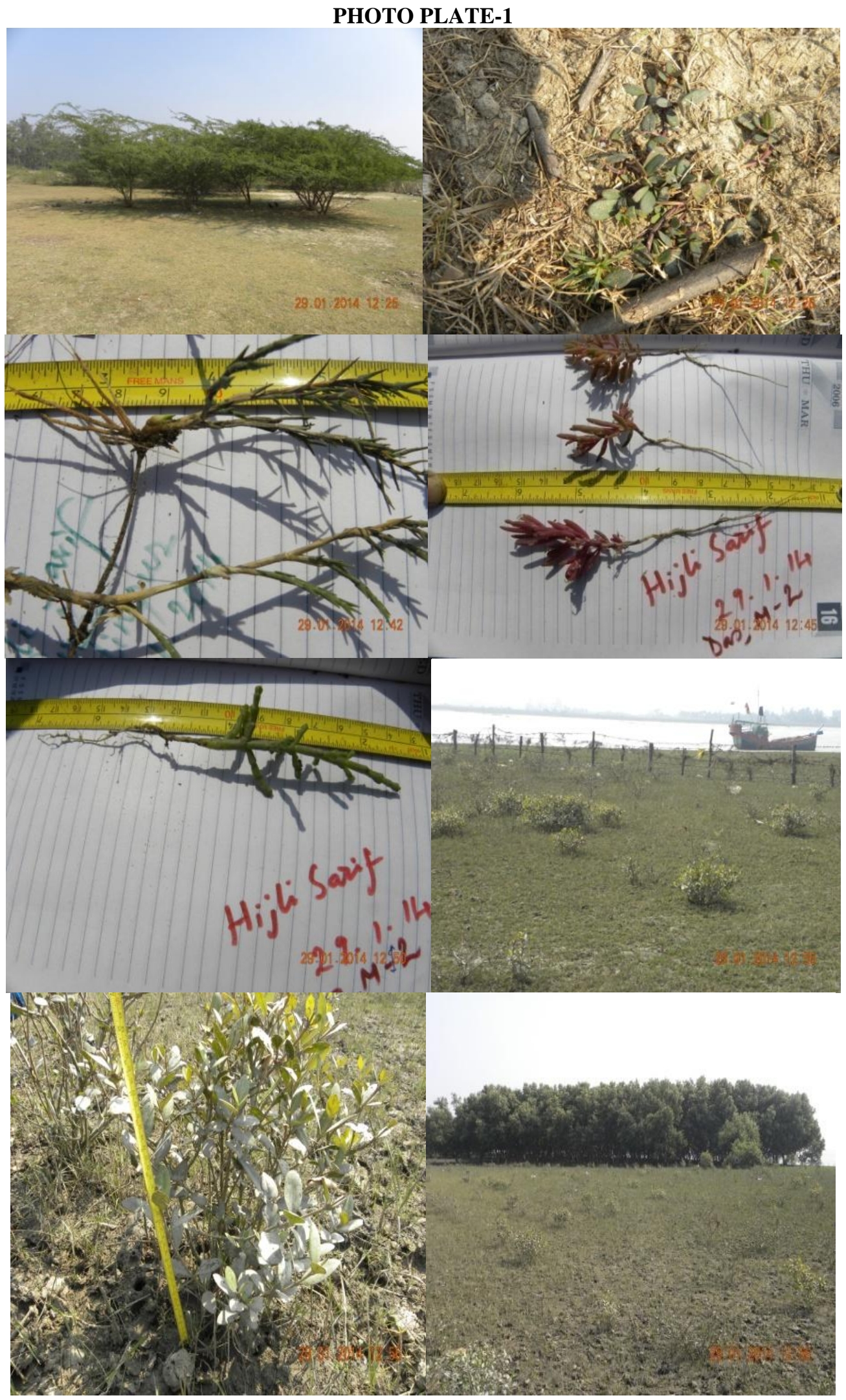




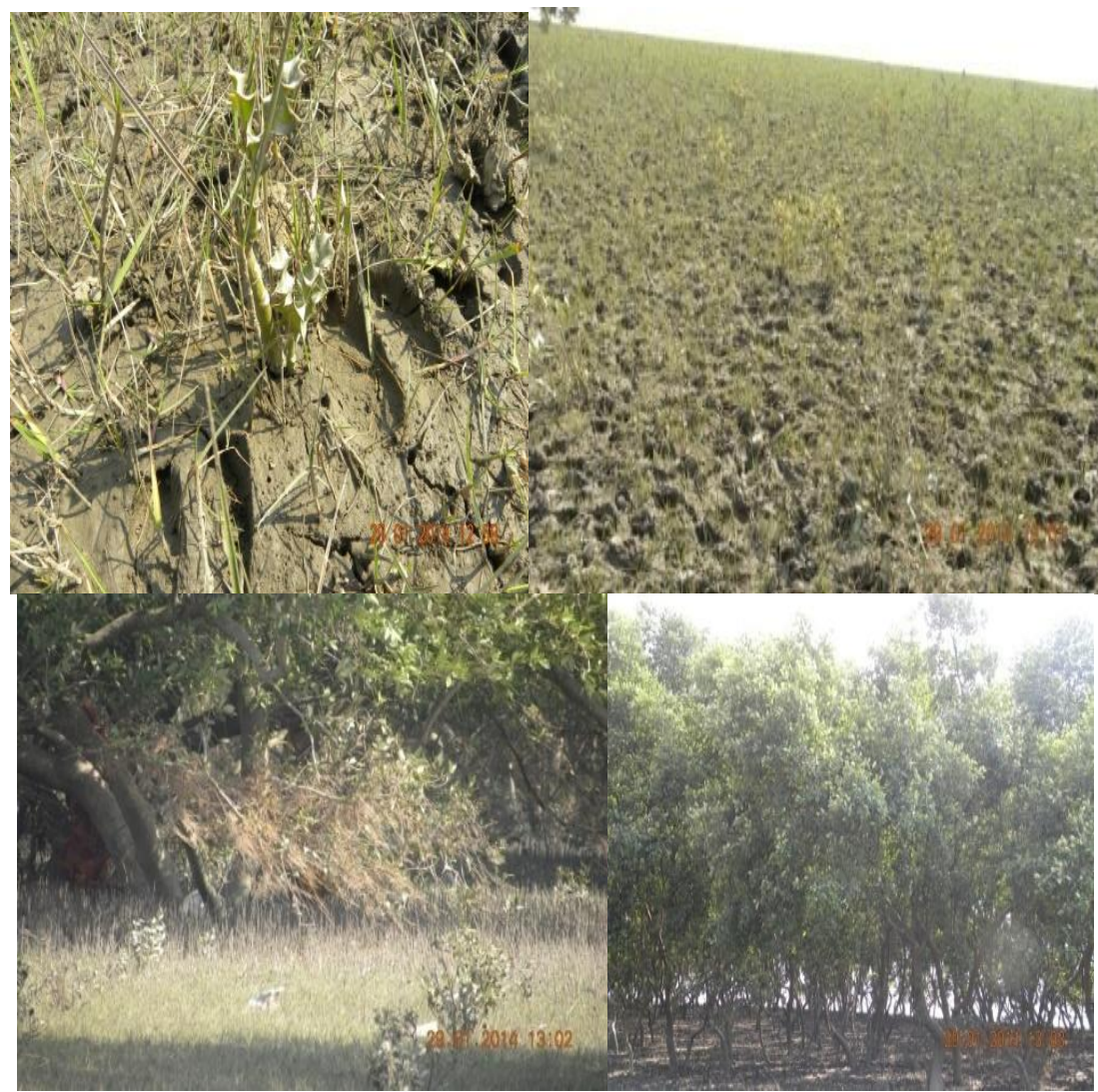

Fig.-1 Community of Prosopis juliflora ; Fig. 2 Sesuvium portulacastrum ; Fig. 3 Hedge community of Aleuropus lagopoides in an open land under low tide (LT) area; Fig. 4 Suaedia maritima; Fig. 5 Salicornia brachiata; Fig. 6 A few years old stand with halophyte plantation by Forest Department with uncontrolled grazing and browsing; Fig. 7 Small element in a plantation stand with disturbed growth; Fig. 8 Old Plantation stand of Halophytes ; Fig. 9 Open Muddy ground with Acanthus ilicifolius (Harkach); Fig. 10 Heavily Grazed land near Shore ; Fig. 11 Halophytic vegetation as Nature's Kidney crabbing huge pollutants ; Fig 12 canopy of Old plantation stand of halophytes with glossy leaves (Figures from top left to top right and similar in way by numbering as $1,2,3, \ldots 4,56, . .7,8,9, . .10,11,12$. in such a way, all photo snaps were taken during field visit by author and co-author in the field)

\section{PHOTO PLATE-2}

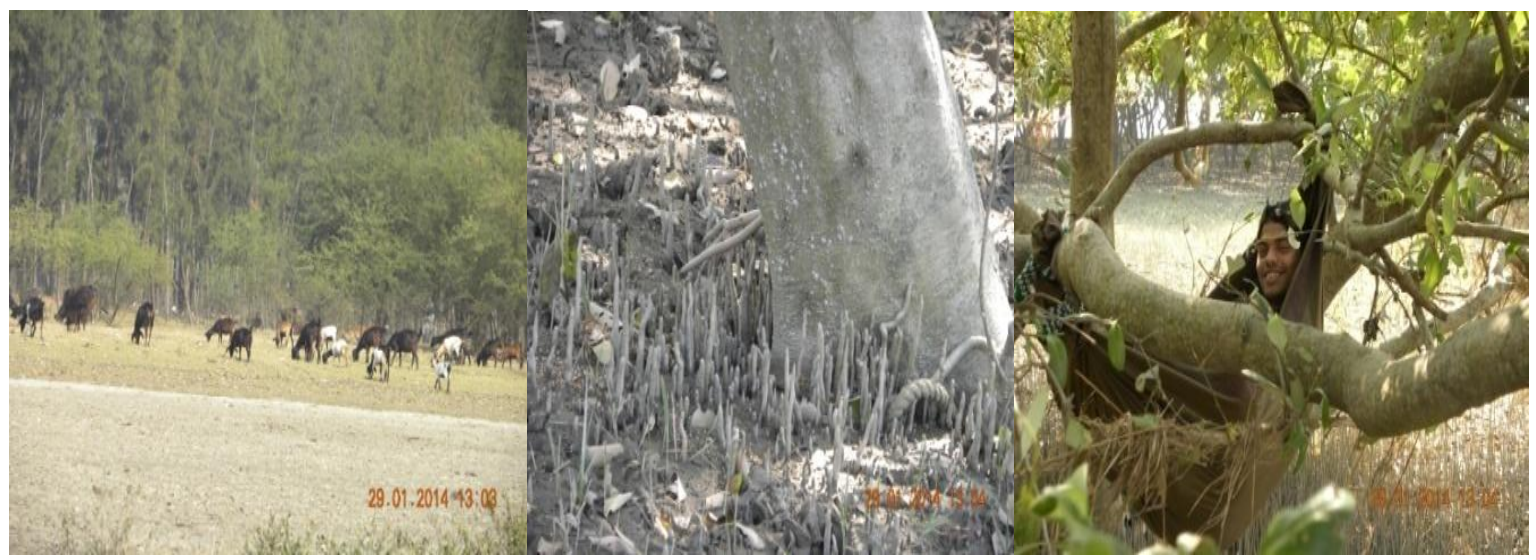




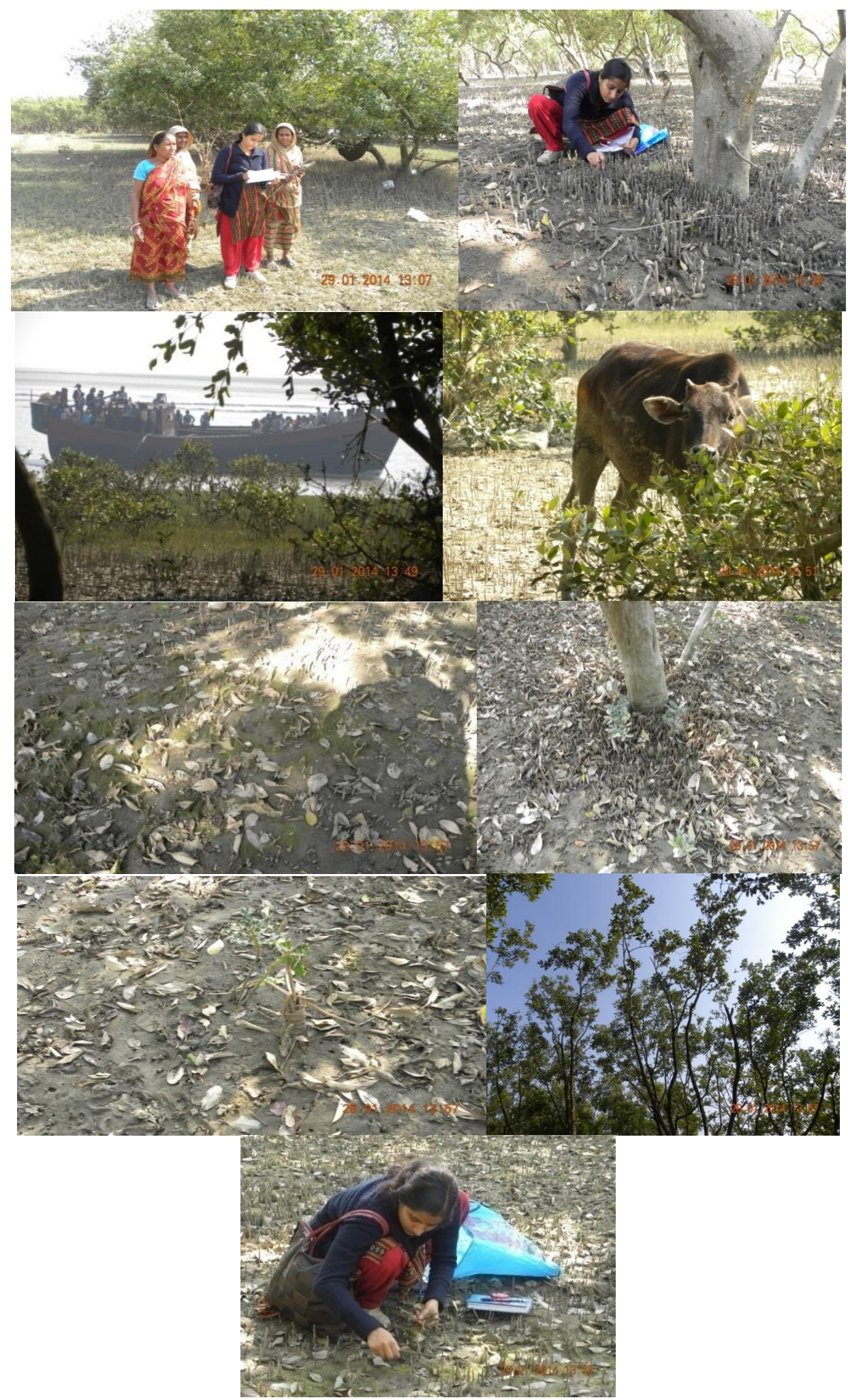

Fig. 13 Grazed animals in open low tide (LT) area near plantation stands; Fig. 14 Pneumetaphores: One kind of areal roots around the tree trunk of Keora at Hijli plantation stand area; Fig. 15 Asleep of Cowboy in a Local Cradle made by bed sheet during afternoon; Fig. 16 Co-author discussing ecological problems of women villagers in field (All are helping to Graze Cattles); Fig. 17 Co-author engaged during study of Pneumetaphores and leaf during data collection in winter; Fig 18. Shoreline with Bulbostylis barbata and sparsely distributed halophyte trees; Fig. 19 Halophytes are grazed by Cow; Fig. 20 Shadow on ground helps to study Canopy cover by graph paper method; 21. Density and abundance of Pneumetaphores are more around the basal area of tree in compare to open floor in an old plantation stand; Fig. 22 Relatively less dispersed area for Pneumetaphores, ; 
Fig. 23 Canopy diversity at different microclimates and stand distance diversity of Halophytes in old plantation; Fig. 24 Co-author collecting Green algae from plantation stand.

PHOTO PLATE-3

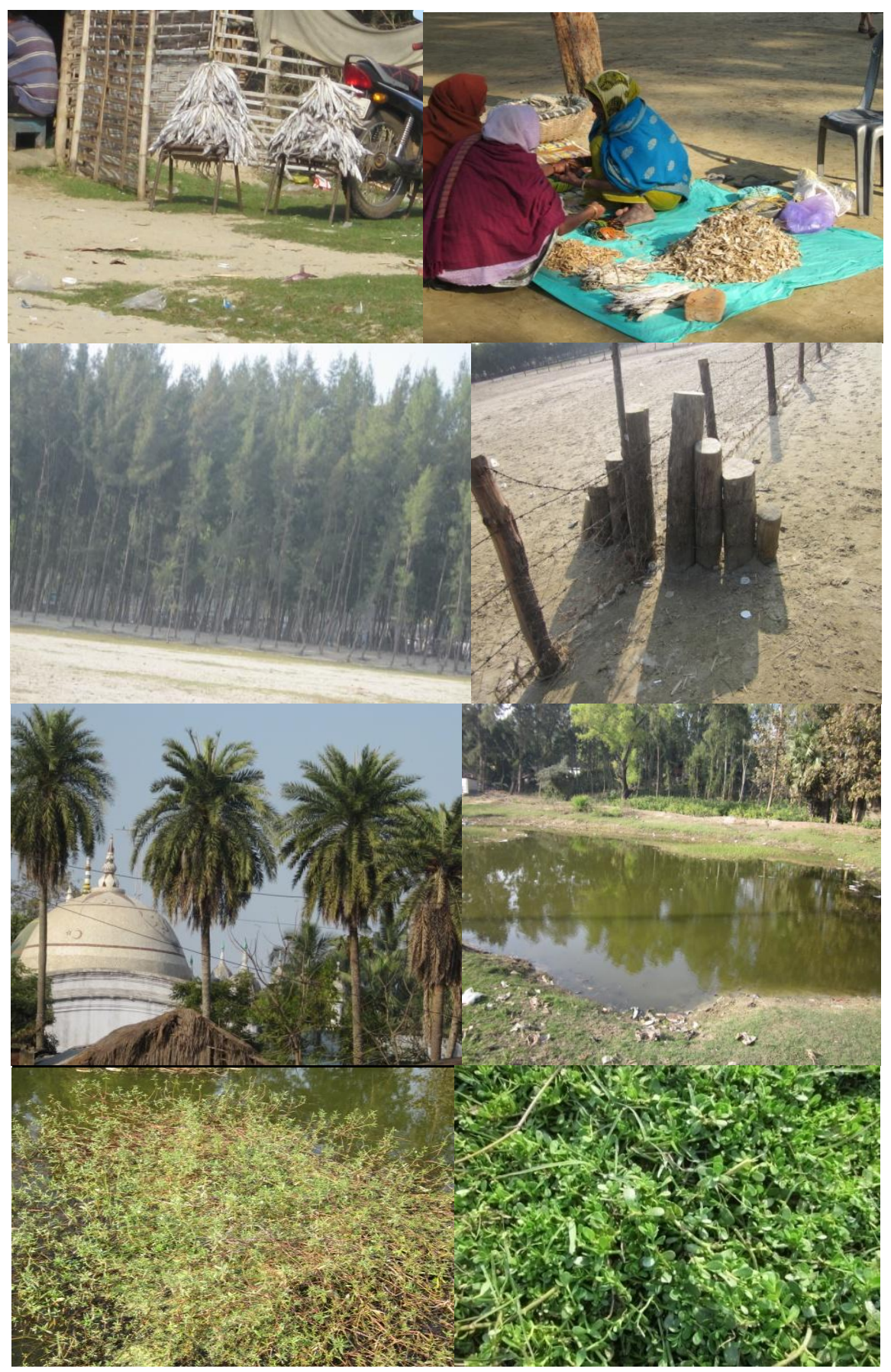




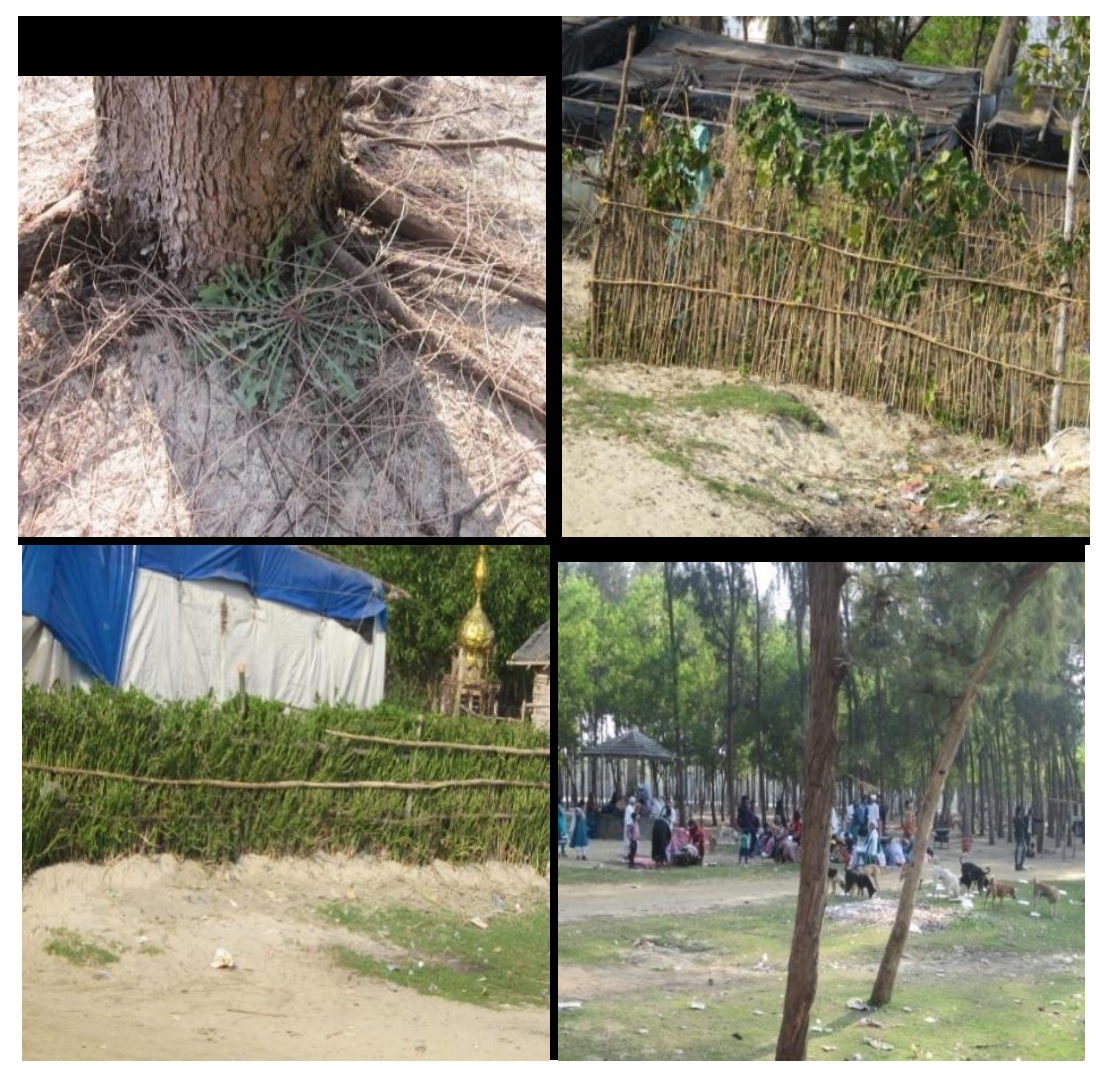

Fig. 25 Dry fishes after processing; Fig. 26 Sellers and Byers at Hijli Sarif area (Baba Saheb er Gargara); Fig. 27 Large Casuarina plantation along the shoreline ; Fig. 28 Stairs to pass across the fencing of Forest Departrnent; Fig. 29 Old Phoenix sylvestris tress near Hijli sarif; Fig. 30 Small pond conserving aquatic plants but now facing pollution of plastic by tourists; Fig. 31 Tillanthera sp. ; Fig. Rotalia sp. 32 ;Fig. 33 Launea sermentosa of Asteraceae near the trunk of old Casuarina tree; Fig. 34 Local fencing made by live Jatropha curcus plants; Fig. 35 Local fencing made by live Pedilanthus tithymeloides plants; Fig. 36 Visitors play a significant role to pollute the environment day by day in a large plantation area which is going to pollute estuary. [Photographs/figures are taken from field by authors and co-authors during field visit at Khejuri-I, and II blocks in Purba Medinipur District of West Bengal, India and photograps taken by Canon and Nikon Camera, Cool pix]

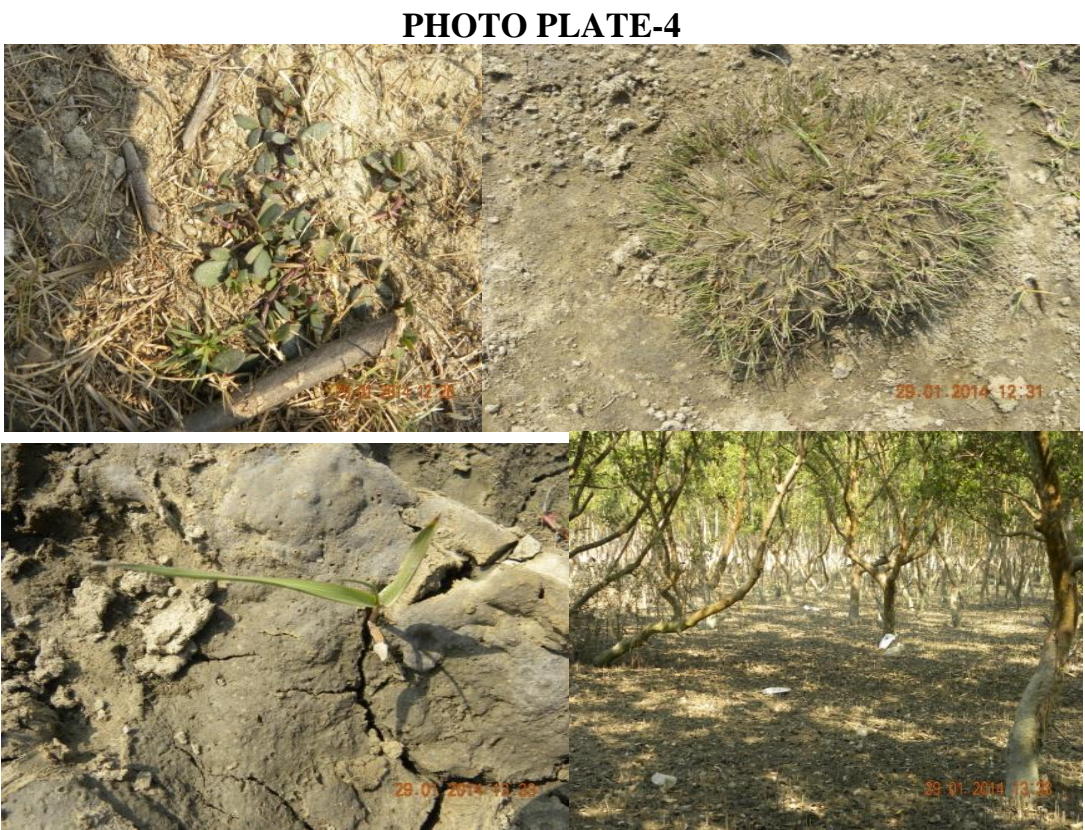




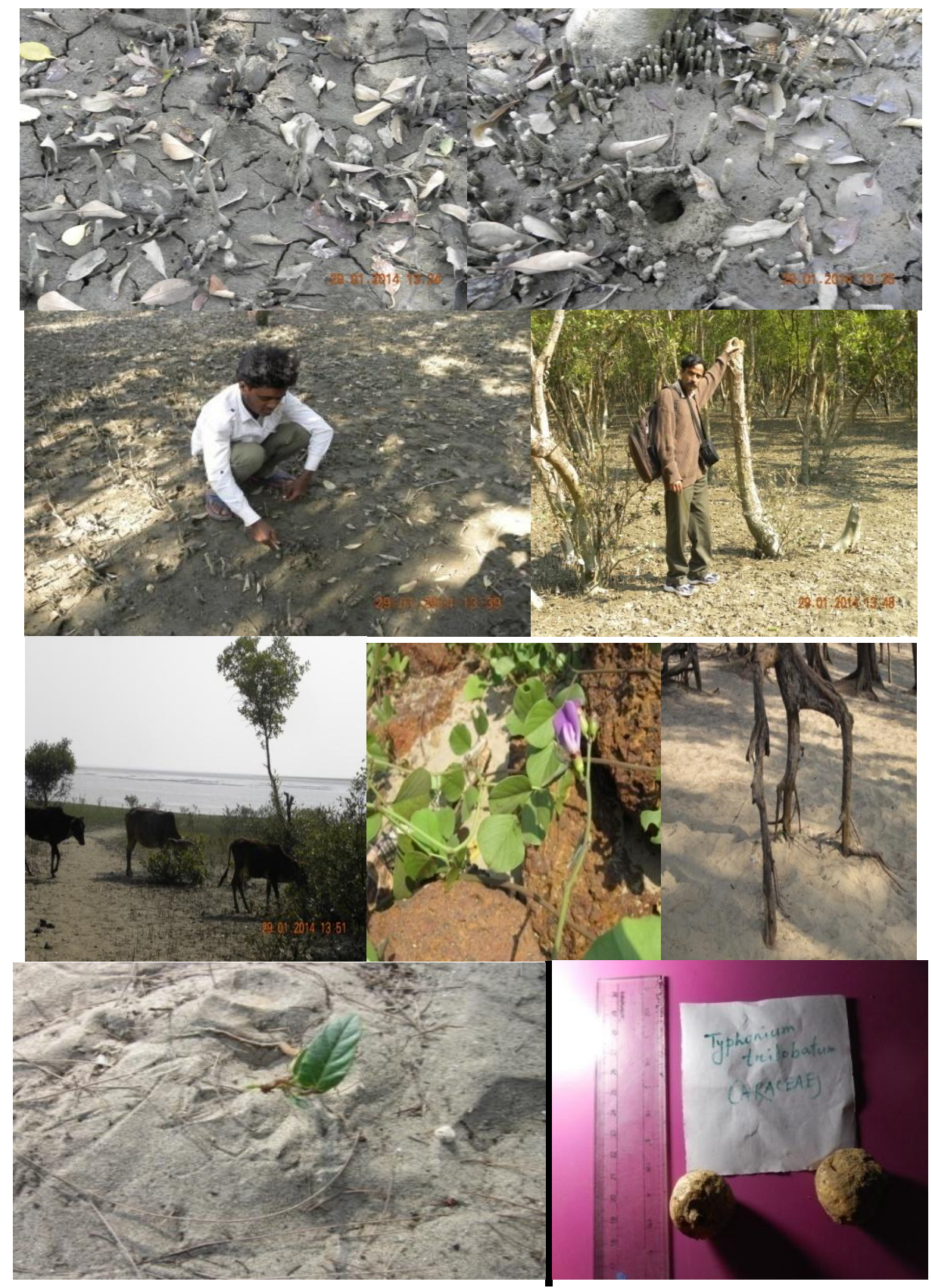

Fig. 37 Sesuvium potulacastrum; Fig. 38 Fimbrystylis ferruginea; Fig. 39 Poteresia coarctata (Seedlings of Dhani grass), Fig. 40 Pollution made by Plastic-Thermocol underneath of Halophytic vegetation is a present problem; Fig. 41 Pattern of leaf fall under the canopy of Keora vegetation; Fig. 42 Crab hole surrounded by numerous aerial roots which protect crab habitat from the settlement of heavy sediment during high tide; Fig. 43 Local driver (Darbesh) helping to collect soil from ground of Keora vegetation; Fig. 44 Author monitoring vegetation and standing crop near a cut pole of Keora tree, which is illegally extracted; Fig. 45 Degraded Vegetation; Fig. 46 Cannavelia rosea (Sw.) DC.(Dune Bean); Fig. 47 Soil erosion causing plant with skeletal stump of Casuarina equisetifolia in Coastal region is a serious problem; Fig. 48 Plant of Tylophora tenuiswhich is a species of Java and Borneo, also found in Sunderbans and Mahanadi tidal swamps, 49. Corm of Typhonium trilobatum-A potent medicinal plant part.

\section{SUMMARY OF THE FINDINGS}

New plantation stand of halophytes are greatly disturbed by locale cattle. The wire fence is greatly damaged by grazers and browsers. So, a few years plantation stand is destroying day by day which need urgent protection to stabilize the bank more vivid. Some patches are so damaged that all the natural floral components are destroyed which loss the local phyto-diversity and afterwards will damage the fauna of the local kind in a great extent. 
Therefore, immediate protection of the same land is required to manage it in near future. Government should take initiative to make the land protected type in near future, so that all the natural species will revive soon by the protection of habitats. This will enforce the ongoing natural process by applying the frame of plantation work and tourists should visit the place following some rules and regulations guided by Ministry of Forests and Environment (MOEF) time to time. A board should be placed in the nearby places and should encourage the people more civilized regarding the pollution and their effects on environment. Seminar and symposium should be organized by Institutions and NGOs regular in basis which can cause the people more literate regarding the problem and prospects of Nature and natural processes. Here, old plantation stand of Keora showed good growth though no setting of seedlings are established, which is another problem in plantation stand (Fig. 42). This is a problem, so incorporation of other species is required to make the plantation stands heterogeneous rather than homogenous. It would generate the admixture of species and species interactions will start which ultimately lead to make the more convenient ecosystem as non-fragile one. The present results of GBH of the species in $10 \mathrm{~m} \times 10 \mathrm{~m}$ quadrat showed homogeneity of the species at old plantation stand rather than heterogeneity (Table 8)

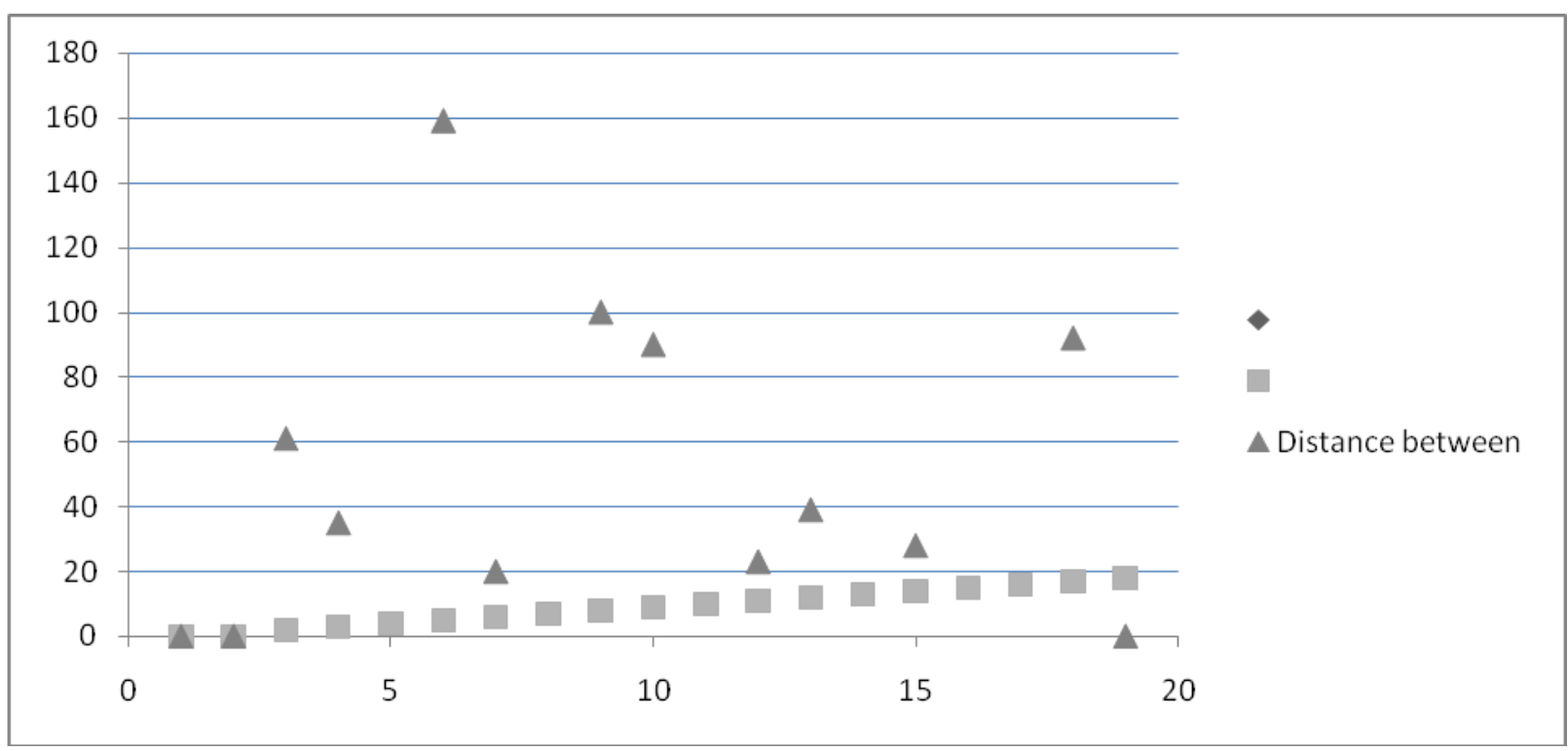

Legend of Fig. 50 Distribution of Halophytic species in Old plantation stand of Hijli Coastal area of Purba Medinipur, West Bengal as per the scattered diagram, data taken from field along with canopy cover to assess the ecological stability before studying succession of natural vegetation, the ground is almost species free though some ground area is filled with Acanthus ilicifolius with low abundance, the major appearance of aerial root like Pneumetaphores are present which boost good features to prevent foreigner even check soil erosion. This is significant to develop climax community. This forest is somehow degrading nearer to banks of the estuary which is always browsed by goats and grazed animals like cows. Another problem is that, some poachers collected wood and woodlots from plantation stand and causing the site degraded. Degradation of soil in ground of forest is discontinuous. It is as because pneumetaphores (Aerial roots) are concentrated near the base of the tree and discontinuous in the space in between trees. So, patches of vegetation and pattern of pneumetaphores are the characteristic of vegetation which cause gradual net formation and therefore protect soil erosion during high tide even during cyclonic waves along with continuous raining. Another problem is that heavy pollution particularly polythene is gathering the forest floor which can degrade the vegetation. Some visitors unknowingly debarking and cutting the trees and branches thereby so diseases come with the fall of balancing of plants and ultimately destroying by pathogenic attack particularly by fungi of different kinds. So, unwanted entry even frequent entry should be checked by the local people even by forest department to make it a sound and environment friendly. Only permitted research and extension may be made with the aid of knowledge may be imposed to challenge the outcome of vegetation and its impact in a society. Exotic alien species likes Lantana camara L. and Eupatorium odoratum Linn. (Chromolaena odorata (Linn.) King \& Robin. ) are not found here which are the source of fuel wood so, incorporation of Casuarina and Acacia should be encouraged which may be the alternate source of the villagers for a varied range of fuel wood collection without any intervention. For overall overview regarding the management for sustenance of life, we must follow the guideline of previous works made by different workers through different projects along the projects allotted for Coastal Zone Management. My research objectives are similar and that is actually linked with the report of 
publications made by authors time to time. As for example, a report of Central Pollution Control board (Anonymous, 2002) suggested that the following works are mandatory for eco-development in coastal area. For the coastal zone, two types of linkages are necessary to achieve inter-sectoral cohesion viz. (i) linkages between the agricultural sector and the industrial sector and (ii) Inter-industry linkages. The planned development of Haldia is apparently considerate to the latter. However, for the vast outlying rural areas including the remotely located places in the Sundarbans region, a linkage relationship is to be established between the agriculture and the industrial sector for a symbiotic growth. Agro-based industry should be set up for reducing the pressure on land and rectifying the increasingly adverse mainland ratio. This could also help in providing the rural population with opportunities for improving their living standards. The said linkage could help provide market for rural skill based products. The frame of work should be made by Scientists, researchers and Government while the force should be impressed by policymakers and politicians to make it a complete and eco-sustainable in near future. So, more and more projects are required to make a comprehensive report of the said area to solve the problem in near future.

\section{ACKNOWLEDGEMENTS}

The authors are thankful to the local people for their assistance during field visit. Authors also acknowledge the persons of the forest department including administrative officers for their cordial help. Librarians of Vidyasagar University; BSI, Kolkata, and different college are acknowledged. Chairpersons of the societies of different journals (online) are acknowledged for literature study. Authority of UGC, ERO, Kolkata, is well acknowledged for permission and financial help. Last but not least thank to persons of the area is due to assistance during sample and data collection.

\section{Journal Papers:}

\section{REFERENCES}

[1] Mandal, M; Dandapath, P K; Bhusan, S. Digha Sankarpur Littoral Tract-A Geographical Case Study, Int. J. Applied \& Humanities and Social Science Invention, 2(4), 2013, 46-54.

[2] Chakraborty, S K. Coastal Environment of Midnapore, West Bengal: Potential Threat and Management, Jour. Coast. Env, 1(1), 2010, 27-40.

[3] Chakraborty, T; Mondal, A K and Parui, S. Studies on the Phytoresources of Coastal dune flora at West Bengal and adjacent Orissa, India, Int. Jour. of Sc. and Nature, 3(4), 2012, 745-752.

[4] Das, D. Pedalium murex L. (Pedaliaceae) - A New Record of Purba Medinipur District to the state of West Bengal, IOSR Jour. Of Business and Management, 13(4), 2013, 54-56.

[5] Ghosh, R B and Das, D. A Preliminary census on pathogenic flora of crop plants in Midnapore District, West Bengal, Indian J. Applied \& Pure Bio., 12(1), 1997, 25-30.

[6] Dey, M K; Hazra A K and Chakraborty, S K. Functional role of Microarthropds in nutrient cycling of Mangrove-estuary ecosystem of Midnapore coast of West Bengal, India, Int. Jour. of Environmental Technology and Management, 12(1), 2010, 67-84.

[7] Banerjee, L K. Conservation of Coastal Plant communities in India, Bull Bot Sur India, 36(1-4), 1994, 160-165.

[8] Corre Jean-Jacques. The Sand Dunes and their vegetation along the Medeterrian coast of France, their likely response to climate change. Landscape Ecology, 6(1\&2), 1991, 65-75.

[9] Desai, K N . Dune Vegetation: need for a reappraisal, Coastin: A Coastal Policy Research Newsltter, $3,2000,6$

[10] Blasco, F. Mangroves of India, Inst. Francais, Pondicherry, Trav. Sect. Sci. Tech., 14, 1975, 1-175.

[11] Banerjee, L K. Ecological Studies on the mangals of Mahanadi estuarine delta, Orissa. Trop. Ecol. , 82, 1987, 117-125.

[12] Dwivedi, S N; Parulekha, A H and Goswami, S C. Ecology of Mangrove swamps of the Mandovi estuary, Goa, India, Proc. Inter. Symp, Manag, Mangroves, Hawai, 1, 1974, 115-194.

[13 ] Mukherjee, B B and Mukherjee, J. Mangroves of Sunderbans, India, Phytomorphology, 28(2),1978, $217-229$.

[14 ] Rao, T A; Sastry, A R K. A n Ecological approach towards classification of Coastal Vegetation of India-I, Strand Vegetation, Ind. For., 98, 1972, 597-607.

[15 ] Rao, T A; Sastry, A R K. An Ecological approach towards classification of Coastal Vegetation of India-II, Estuarine Vegetation, Ibid. 100, 1974, 438-452.

[16] Sanyal, P; Banerjee, L K and Chowdhury, M K. Dancing Mangals of Indian Sunderbans, Jour. Ind. Soc. Coastal Agri. Res., 2(1), 1984, 10-16.

[17 ] Sidhu, S S. Studies on Mangroves, National Acad. Sci., Allahabad, India, 1960, 111-112.

[18 ] Thothatri, K. Studies on the mangroves of Peninsular India vs. the Andaman and Nicobar Islands, Bull. Bot. Surv. India, 23, 1981, 151-154.

[19] Waheed Khan, M A. Ecological studies on the mangrove forests in India, In: Proc. Mangs. Symposium, Faridabad, 97-113, 1959.

[20 ] Walson, J G. Mangrove forest of the Malay peninsula, Malayan For. Rec, 6, 1928, 1-275.

[21] Subhanian, H; Motamad, N; Ferdous, J R; Razavi, K; Niknam, V and Komatsu, S. Salt stress response of a Halophytic grass Aleuropus lagopoides (L.) Trin. and subsequent recovery, Russian Jour. of Plant Physiology, 57(6), 2010.

[22] Jha, B ; Gontia, I and Hartmann, A. The roots of halophyte Salicornia brachiata are a source of new halotolerant diazotrophic bacteria with plant growth-promoting potential, Plant Soil, Regular article, 2011, 1-13.

[23] Ahmed, M Z; Gilani, S A , Kikuchi, A and Gulzar, S ; Khan, M A and Watanabe, K N. Population diversity of Aleuropus lagopoides: A potential cash crop for saline land, Pak. J. Bot., 43(1), 2011, 595-605.

[24] Das, D and Ghosh, R B. Mangroves and other phanerogams growing at Nayachar, Haldia, Midnapore, West Bengal, Environment and Ecology, 17(3), 1999, 725-727.

\section{Books:}

[25] Bandyopadhyay, K B. Amader Rajya (Bengali Version for West Bengal), Kishore Gyan Vigyan Prakashani, Spectrum Offset, Kolkata-37, 2009, pp. 76.

[26] Anonymous. Flora of West Bengal, Vol.-1, 1997, Flora of India, Series-2, BSI, Kolkata. 
[27] Anonymous. Medicinal Plant Resources of South West Bengal, Vol.-I, 2005, Research Wing, Directorate of Forests, Govt. of W.B.

[28] Anonymous. Medicinal Plant Resources of South West Bengal, Vol.-II, 2010, Research Wing, Directorate of Forests, Govt. of W.B.

[29] Bennet, S S R. Name changes in Flowering Plants of India and Adjoining Regions, Triseas Publishers, 1987, Dehra Dun.

[30] Duthie, J F. Flora of Upper Gangetic plains, Vol. I, II, 1960, Botanical Survey of India, Calcutta.

[31] Haines, H H. The Botany of Bihar and Orissa, Vol. I-IV,(1921-1925), BSI, Calcutta.

[32] Hooker, J D. Flora of British India, Vol. 1-7, (1892-1897), BSI, Calcutta.

[33] Jain, S K and Rao, R. R. A Hand book of Field Herbarium Methods, Oxford and IBH Publishing Company, 1977, New Delhi.

[34] Kirtikar, K R and Basu B D. Indian Medicinal Plants, I-IV, International Book Distributors, 1918, Dehra Dun.

[35] Prain, D. Bengal Plants (Vol-I, II), Bishen Singh and Mahendra Pal Singh, 1963, Dehradun. Revised Edn, BSI, Kolkata.

[36] Roy S B; Mahapatra, T and Yadav, G. Forest Protection and management by communities in Midnapore District, West Bengal Published in the Book "Joint Forest Management and Community Forestry in India-An Ecological and Institutional Assessment" Edited by N H Ravindranath, K S Murali and K C Malhotra, Oxford IBH Pub. Co. Pvt. Ltd.,2000.

[ 37] Banerjee, L K; Sastry, A R K and Nayar, M P. Mangroves of India-Identification Manual, BSI, Kolkata-1, 1989.

[38] Rimer, D N. Inroduction to Fresh water vegetation, AVI Publishing Company, INC, Westport, Connecticut, 1984.

[ 39] Naskar, K and Guha Bakshi, D N. Mangroves swamps of the Sunderbans, Naya Prakash, Calcutta, 1987.

[40] Sidhu, S S. Studies on Mangroves, National Acad. Sci., Allahabad, India, 11-112, 1960.

[41] Anonymous. Productivity of an Anthocnemum indicum dominated coastal salt marsh at Karachi, Pakistan, Chapter-II of book, Biology of Salt Tolerant Plants, Editted by Khan, M A and Ungar, I A, 1995.University of Karachi.

[42] Anonymous. Medicinal plant Resources of Kolkata, A Photo Guide, Research Circle, Forest Directorate, Govt. of West Bengal, 2012, pp.1-200.

[43] Maity, P K and Maity, P. Biodiversity, Perception, Peril and Preservation, PHI Learning Pvt. Ltd, Eastern Economy Edition, New Delhi, 1

Thesis:

[44] Das, D. 2007. Vegetation Ecology of Forests of Southwest Bengal with special reference to Non-Timber Forest Produce (NTFPs) Productivity, Ph.D Thesis, awarded the degree from Vidyasagar University, Midnapore, West Bengal (from CNH, BSI worker, Kolkata).

Related Articles (Manual and Web articles):

[45] Anonymous Chapter-II, General Description of the District, Website.

[46] Rao, R R and Sharma, B D. A Manual for Herbarium Collections, Botanical Survey of India, Brabourne Road, Kol-1, 1990.

[47] Anonymous, ICMAM Project Directorate, Chennai, Sensitive Coastal Marine Areas of India, 2007, 20-21. 